\title{
Generalized Sensitivities and Optimal Experimental Design
}

\author{
MA/ST 810, FALL, 2009 \\ H. T. Banks and M. Davidian \\ Center for Research in Scientific Computation \\ Center for Quantitative Sciences in Biomedicine \\ North Carolina State University
}

November 8, 2009 
- Consider problem of estimating parameter $\theta$ using weighted least squares criterion for given data $y$

$$
J_{d}(y, \theta)=\sum_{i=1}^{n} \frac{1}{\sigma(t)^{2}}\left(y\left(t_{i}\right)-f\left(t_{i}, \theta\right)\right)^{2}
$$

- Introduce an abstract framework involving generalized measurement procedures characterized by probability measures

- Take an optimal design perspective, general premise: in data, information content may vary considerably-some measurements may be much more informative than others

- Concepts of traditional and generalized sensitivity functions-use to develop strategy to determine "optimal" data-based on time evolution of sensitivity fens and of that of condition number of Fisher information matrix 


\section{PROBLEM FORMULATION}

- Assume that we have a dynamical system which models some physical, sociological or biological phenomenon, e.g.,

$$
\begin{aligned}
& \dot{x}(t)=\mathcal{F}(t, x(t), \theta), \quad x(0)=x_{0}(\theta), \\
& \eta(t)=h(t, x(t), \theta), \quad t \in[0, T],
\end{aligned}
$$

where $x(t) \in \mathbb{R}^{N} \eta(t) \in \mathbb{R}^{M}$ is the vector of observable or measurable outputs, $\theta \in \mathbb{R}^{p}$ with $\mathcal{F}$ and $h$ are mappings $\mathbb{R}^{1+N+p} \rightarrow \mathbb{R}^{N}$ and $\mathbb{R}^{1+N+p} \rightarrow \mathbb{R}^{M}$, respectively.

- Mathematical model:

$$
\eta(t)=f(t, \theta), \quad 0 \leq t \leq T,
$$

where $f$ is defined as $f(t, \theta)=h(t, x(t, \theta), \theta)$. 
- Can also treat dynamical systems other than ordinary differential equations such as (1); for instance delay systems or partial differential equations may in principle also be treated with formulation. That $\eta$ is the vector of observable/measurable outputs of the physical, sociological or biological system implicitly assumes that we have measurements $y(t)$ for $\eta(t)$ at time instances in some subset of $[0, T]$. For the purpose of this discussion we assume that we have a single output system, i.e., we have $M=1$. 
There are typically two problems of interest in the context of a model of type (1)-(2):

- The forward or the direct problem, which directly manifests the dependence of the output variable $\eta$ on the parameter vector $\theta$. An important task is to identify those parameters to which the model output is the most/least sensitive.

- The inverse problem or parameter estimation problem, which consists of estimating the parameter vector $\theta$ from a set of time observations $y$ of the output variable $\eta$. 
- Sensitivity of model outcome to various parameters provides important information on where to interrogate or observe system in order to optimize outputs or to avoid undesirable system outcomes- very important questions in simulation studies and traditional sensitivity functions (TSF) frequently used to investigate

- to solve parameter estimation problem one requires (i) set of measurements of quantity in real system which corresponds to model output, (ii) an error functional for comparison of model output and data (iii) numerical algorithm in order to obtain estimates for parameter vector $\theta$ as minimizers of an error functional, such as for instance least squares or maximum likelihood, etc. 
- In order to ascertain quality of parameter estimates one computes standard errors and establishes confidence intervals (see $[7,11,17])$ - obvious that quality of the results depends on all three factors involved, namely quality of mathematical model, the uncertainty in the data, and the performance of estimation algorithm.

- Consider problem of estimating parameter vector $\theta$ from an optimal design perspective-premise is that information content on the parameters $\theta$ may vary considerably from one time measurement to another.

- Intuitively, data with higher information content wrt parameters to be estimated should yield more accurate estimates-goal: to develop and illustrate mathematical tools which can be used to specify duration, number and distribution of times for sampling data in order to obtain estimates with improved accuracy. 
- Assume dynamics of real system such that time behavior of output $\eta$ for some nominal parameter vector $\theta_{0}$ coincides with the time behavior of corresponding quantity of the real system ( existence of a "true" parameter assumption typically encountered in statistical analysis) - a measurement taken at any time $\tilde{t}$ is given by $y(\tilde{t})=\eta\left(\tilde{t}, \theta_{0}\right)$.

- Use (weighted) sum of squares (WLS) of differences between measurements and outputs at the chosen measurement times. As a measure of the quality (which we want to optimize), of the estimates, use standard errors of estimates and/or other design criteria. 
- Specifically want to address the following important questions in parameter estimation problems when considering models of type (1)-(2):

1. How do we choose the duration $T$ of the experiment and the number $n$ of measurements in the interval $[0, T]$, in order to obtain estimates of a high quality for $\theta_{0}$ ?

2. Once the duration $T$ and the number $n$ of measurements are established, what is the optimal sampling distribution in the interval $[0, T]$ in order to obtain most accurate estimates? 
- First question pertains to the minimum time interval $[0, T]$ and to the minimal number of measurements taken in this time interval such these measurements provide enough information on $\theta_{0}$ in order to achieve an acceptable accuracy in the estimates.

- Second question constitutes the main topic of the optimal experimental design literature, and the traditional way to answer it is to find sampling distributions for measurements which optimize some specific design criterion. 
Criterion typically a given function of Fisher information matrix-this matrix depends on nominal parameter $\theta_{0}$, which in practice is unknown-to overcome this difficulty one usually substitutes an initial guess $\theta^{*}$ for $\theta_{0}$-resulting designs are called locally optimal designs. Of the numerous design strategies found in the literature we mention following three among the more popular ones:

a) D-optimal design, which requires one to maximize determinant $\operatorname{det} F(\hat{\theta})$ of the Fisher information matrix-largely accepted in literature because of its appealing geometrical interpretation involving asymptotic confidence regions (which are ellipsoids) for a maximum likelihood estimate $\hat{\theta}$ of $\theta$. One can show that an optimal design with respect to this criterion yields approximately a minimal value for the volume of the error ellipsoid of the estimates;

b) c-optimal design,for $c$ a given vector in $\mathbb{R}^{p}$, requires one to 
minimize the variance of the linear combination $g(\theta)=c^{\top} \theta$ of the parameters to be estimated. Up to a multiplicative constant, the asymptotic covariance of the maximum likelihood of $g$ is given by $c^{\top} F^{-1}(\hat{\theta}) c$, and an optimal design wrt this criterion minimizes this variance. In the particular case when $c$ is the $i$-th unit vector, i.e., $c=e_{i}$, the $c$-optimal design minimizes the variance of the least squares estimate for the $i$-th parameter $\theta_{i}$;

c) E-optimal design, requires one to maximize smallest eigenvalue $\lambda_{\min }(F(\hat{\theta}))$ of Fisher information matrix-equivalent to minimizing the maximum eigenvalue $\lambda_{\max }\left(F^{-1}(\hat{\theta})\right)$ of the inverse $F^{-1}$ (the covariance matrix), or to minimizing the worst variance among all estimates $c^{\top} \hat{\theta}$ for the linear combinations $c^{\top} \theta$ with a given norm $c^{\top} c=1$. Geometrically, the $E$-optimal design minimizes the maximum diameter of the asymptotic confidence ellipsoids for $\theta$. 
Practical importance of the issues addressed by the previous two questions is

- Obvious for experiments where the cost of measurements (invasive procedures, expensive assays and technology, necessity of highly trained personnel, etc.) is high and where one wishes to avoid running the experiments longer than necessary and/or taking more measurements than needed.

- Latter point also can be important in cases where too many measurements may change the dynamics of the system (for instance, when measurements require one to take repeated blood or tissue samples). 
- Introduce a general setting which involves idealized measurement procedures-setting will allow one to prove the existence of idealized measurement procedures as solutions

- Although in general these idealized measurement procedures cannot be realized, may be achieved as limit of concrete measurement procedures.

- Any idealized measurement procedure can be approximated arbitrarily closely by discrete (and therefore realizable) measurement procedures

- Strategy based on time evolution of sensitivity functions (TSF, GSF) and that of condition number of Fisher information matrix - To our knowledge, GSFs introduced first in this connection by Thomaseth and Cobelli, see $[8,12,35])$ 
- Use the Verhulst-Pearl logistic population model as an example to illustrate our results. This example is widely used in the research literature, and has an analytical solution, few parameters, and well-known dynamics (see [8, 11, 28]). This logistic model, which approximates the size of a saturation limited population over time, is described by the differential equation

$$
\dot{x}(t)=r x(t)\left(1-\frac{x(t)}{K}\right), \quad x(0)=x_{0},
$$

where the constants $K, r$ and $x_{0}$ represent the carrying capacity of the environment, the intrinsic growth rate in the population and the initial population size, respectively. 
- one typically treats the initial condition also as a parameter, the parameter vector we wish to estimate is $\theta=\left(K, r, x_{0}\right)^{\top} \in \mathbb{R}^{3}$. The analytical solution of (3) is given by

$$
x(t)=\frac{K}{1+\left(K / x_{0}-1\right) e^{-r t}},
$$

and this approaches the steady state $x \equiv K$ as $t \rightarrow \infty$ (see Figure 1(a)) and will be taken as the output of the system, i.e., the right side of equation (4) defines the function $f(t, \theta)$ in this case. 


\section{A General Formulation of the Parameter Estimation Problem}

\section{Measurement Procedures}

Given $T>0$ we assume that we can take measurements for any $t \in[0, T]$. Following standard statistical theory, we consider

$$
y(t)=f\left(t, \theta_{0}\right)+\varepsilon(t), \quad t \in[0, T],
$$

as the realization model for the observation process, where $\theta_{0}$ is the "true" or nominal parameter vector, which is unknown to us. The nonlinear regression function $f$ is assumed to be continuous and twice differentiable with respect to the parameters $\theta$. (Later shall need assumption that the time behavior of output $\eta$ coincides with time behavior of the corresponding quantity of the real system not 
only for the nominal parameter vector $\theta_{0}$ but for all $\theta$ in a neighborhood of $\theta_{0}$.)

The function $y$ which gives measurements at any time $t \in[0, T]$ is a realization (sample path) of a stochastic process (the statistical model)

$$
Y(t)=f\left(t, \theta_{0}\right)+\mathcal{E}(t),
$$

which governs measurement process. As usual $f\left(t, \theta_{0}\right)$ is (deterministic) model output corresponding to the true parameter $\theta_{0}$ and $\mathcal{E}$ is a noisy random process for measurement errors.

$$
\begin{aligned}
E(\mathcal{E}(t)) & =0, \quad t \in[0, T], \\
\operatorname{Var} \mathcal{E}(t) & =\sigma^{2}(t), \quad t \in[0, T], \\
\operatorname{Cov}(\mathcal{E}(t) \mathcal{E}(s)) & =\sigma(t) \sigma(s) \delta(t-s), \quad t, s \in[0, T],
\end{aligned}
$$

where the Dirac function $\delta$ satisfies $\delta(\tau)=1$ for $\tau=0$ and $\delta(\tau)=0$ for $\tau \neq 0$. 
Let $m(t)$ denote the measurement density at time $t \in[0, T]$, i.e.,

$$
\int_{s}^{t} m(\tau) d \tau
$$

is the number of measurements in the interval $[s, t], s<t$. In order to define the least squares error $J(y, \theta)$ for a given measurement procedure $y(\cdot)$, and for $\theta$ in a neighborhood of $\theta_{0}$, we start with the weighted error for one measurement at time $t$, which is given by $\sigma(t)^{-2}(y(t)-f(t, \theta))^{2}$, i.e., we put more weight on measurements where the variance of the measurement error is smaller. 
Next we choose mesh points $t_{i}=i \Delta t, i=0, \ldots, n$, with $n=T / \Delta t$. We assume that $\Delta t>0$ is chosen sufficiently small so that the number of measurements in the time intervals $\left[t_{i}, t_{i}+\Delta t\right]$, $i=0, \ldots, n-1$, is given by $m\left(t_{i}^{*}\right) \Delta t$ and the average weighted error on $\left[t_{i}, t_{i}+\Delta t\right]$ by $\sigma\left(t_{i}^{* *}\right)^{-2}\left(y\left(t_{i}^{* *}\right)-f\left(t_{i}^{* *}, \theta\right)\right)^{2}$ for some $t_{i}^{*}, t_{i}^{* *} \in\left[t_{i}, t_{i}+\Delta t\right]$. Here we assume for the moment that $m$ and $\sigma$ are continuous. Then the weighted errors in the intervals $\left[t_{i}, t_{i}+\Delta t\right]$ are given by

$$
\frac{m\left(t_{i}^{*}\right)}{\sigma\left(t_{i}^{* *}\right)^{2}}\left(y\left(t_{i}^{* *}\right)-f\left(t_{i}^{* *}, \theta\right)\right)^{2} \Delta t, \quad i=0, \ldots, n-1 .
$$

Taking $\Delta t \rightarrow 0$ we obtain

$$
J(y, \theta)=\int_{0}^{T} \frac{m(t)}{\sigma(t)^{2}}(y(t)-f(t, \theta))^{2} d t .
$$


The integral can be viewed as an integral with respect to an absolutely continuous measure $P$ on $[0, T]$ with density $m(t)=\frac{d P}{d t}(t)$. This motivates us to consider, more generally, error functionals of the form

$$
J(y, \theta)=\int_{0}^{T} \frac{1}{\sigma(t)^{2}}(y(t)-f(t, \theta))^{2} d P(t),
$$

where $P$ is a general measure on $[0, T]$. Since we shall obtain the parameter estimate $\hat{\theta}$ by minimizing $J(y, \theta)$ for $\theta$ in a neighborhood of $\theta_{0}$, we can, without restriction of generality, assume that $P$ is a probability measure on $[0, T]$. 
If, for points $t_{1}<\cdots<t_{n}$ in $[0, T]$, we take

$$
P_{d}=\sum_{i=1}^{n} \delta_{t_{i}},
$$

where $\delta_{a}$ denotes the Dirac delta distribution with support $\{a\}$, we obtain

$$
J_{d}(y, \theta)=\sum_{i=1}^{n} \frac{1}{\sigma\left(t_{i}\right)^{2}}\left(y\left(t_{i}\right)-f\left(t_{i}, \theta\right)\right)^{2},
$$

which is the weighted least squares cost functional for the case where we take a finite number of measurements in $[0, T]$. Of course, the introduction of the measure $P$ allows us to change the weights in (9) or the weighting function in (7). For instance, if $P$ is absolutely continuous with density $m(\cdot)$ the error functional $(7)$ is just the weighted $L^{2}$-norm of $y(\cdot)-f(\cdot, \theta)$ with weight $m(\cdot) / \sigma(\cdot)^{2}$. 


\section{Least Squares Estimates}

In order to estimate $\theta_{0}$, we use a weighted least squares procedure, i.e., the parameter estimate $\hat{\theta}$ is an admissible parameter vector which minimizes the cost functional (7) with given measurements $y(\cdot)$,

$$
\hat{\theta}=\underset{\theta}{\operatorname{argmin}} J(y, \theta),
$$

Since the specific data $y$ is a realization of the stochastic process $Y$, we consider $\hat{\theta}$ as a realization of a random variable $\hat{\Theta}$, which usually is called the weighted least squares estimator for the parameter estimation problem. Symbolically we rewrite (10) as

$$
\hat{\Theta}=\underset{\theta}{\operatorname{argmin}} J(Y, \theta) .
$$

An important question to address is how the uncertainty in the measured data propagates into the parameter estimates. This is 
equivalent to determining the statistical properties of the estimator $\hat{\Theta}$ based on those of $Y$. Using the linearization argument presented in detail below, we obtain that to a first order approximation we have the distribution results

$$
\hat{\Theta} \sim \mathcal{N}_{p}\left(\theta_{0}, \Sigma_{0}\right)
$$

i.e., $\hat{\Theta}$ is approximately a normally distributed $p$-dimensional random vector with expected value $\theta_{0}$ and covariance matrix $\Sigma_{0}$, where $\Sigma_{0}$ is the inverse of the Fisher information matrix (28). In order to quantify the accuracy of specific estimates $\hat{\theta}$, and to compare between different sampling strategies, we use the classical approach in asymptotic statistical analysis, and compute the standard errors $S E_{k}$ given by

$$
S E_{k}=\sqrt{\left(\Sigma_{0}\right)_{k k}}, \quad k=1,2, \ldots, p .
$$


The standard errors can be used to compute the confidence intervals, which give further insight about the quality of the parameter estimates obtained.

It is important to note that the matrix $\Sigma_{0}$ depends explicitly on the measure $P$ (see (28) in Section 39). Changing $P$ affects $\Sigma_{0}$, which in turn controls the accuracy of the least square estimates (10). At this point the potential use of the measure $P$ in (7) as an optimal design tool becomes apparent. For a given class of measures $P$ on the interval $[0, T]$ we want to find the one which yields the most accurate parameter estimates. 


\section{Linear Regression - Distributed Measurements}

In this subsection we consider the case where the output model (2) is linear in $\theta$, i.e., we have

$$
\eta(t)=G(t) \theta, \quad 0 \leq t \leq T, \theta \in \mathbb{R}^{p},
$$

where

$$
G(t)=\left(g_{1}(t), \ldots, g_{p}(t)\right) \in \mathbb{R}^{1 \times p}, \quad 0 \leq t \leq T,
$$

with functions $g_{i}:[0, T] \rightarrow \mathbb{R}, i=1, \ldots, p$. We assume that the measurements are given by

$$
y\left(t, \theta_{0}\right)=G(t) \theta_{0}+\epsilon(t), \quad 0 \leq t \leq T,
$$

where $\theta_{0}$ is the true or nominal parameter vector and $\epsilon(\cdot)$ is a realization of the noise process $\mathcal{E}(\cdot)$ which is assumed to satisfy (6). The parameter estimation problem would require one to solve the equation $G(t) \theta=y\left(t, \theta_{0}\right)$, which in general does not have a solution 
because of the noise term in $y\left(\cdot, \theta_{0}\right)$. Therefore we consider the residual $y\left(\cdot, \theta_{0}\right)-G(\cdot) \theta$ and attempt to find the estimate $\hat{\theta}=\hat{\theta}\left(\theta_{0}\right)$ as an element in $\mathbb{R}^{p}$ which minimizes a weighted means square of this residual, i.e.,

$$
\hat{\theta}=\underset{\theta \in \mathbb{R}^{p}}{\operatorname{argmin}} \int_{0}^{T} \frac{1}{\sigma^{2}(t)}\left(y\left(t, \theta_{0}\right)-G(t) \theta\right)^{2} d P(t) .
$$

We introduce the weighted $\mathrm{L}^{2}$-space $L_{w}^{2}=L_{w}^{2}(0, T)$ of all $P$-measurable functions $u:[0, T] \rightarrow \mathbb{R}$ such that $u / \sigma$ is square integrable on $[0, T]$ with respect to the measure $P$ and define the operator $\mathcal{G}: \mathbb{R}^{p} \rightarrow L_{w}^{2}$ by the right side of (12) assuming that $g_{i} \in L_{w}^{2}, i=1, \ldots, p$. Then we can write (14) as

$$
\hat{\theta}=\underset{\theta \in \mathbb{R}^{p}}{\operatorname{argmin}}\left\|y\left(\cdot, \theta_{0}\right)-\mathcal{G} \theta\right\|_{L_{w}^{2}}^{2} .
$$


This is a standard minimum norm problem [29] in the Hilbert space $L_{w}^{2}$ which always has solutions $\hat{\theta}$. These solutions satisfy the normal equations

$$
\mathcal{G}^{*} \mathcal{G} \hat{\theta}=\mathcal{G}^{*} y\left(\cdot, \theta_{0}\right),
$$

where $\mathcal{G}^{*}: L_{w}^{2} \rightarrow \mathbb{R}^{p}$ is the adjoint operator of $\mathcal{G}$ defined by $\langle\mathcal{G} \theta, u\rangle_{L_{w}^{2}}=\left\langle\theta, \mathcal{G}^{*} u\right\rangle_{\mathbb{R}^{p}}$ for all $\theta \in \mathbb{R}^{p}$ and all $u \in L_{w}^{2}$. By the general theory of generalized or pseudo inverses and minimum norm problems in Hilbert spaces [29, Chapter 6] one can under appropriate conditions obtain a unique $\hat{u} \in$ range $\mathcal{G}$ such that $\left\|y\left(\cdot, \theta_{0}\right)-\hat{u}\right\|_{L_{w}^{2}}=\min _{u \in L_{w}^{2}}\left\|y\left(\cdot, \theta_{0}\right)-u\right\|_{L_{w}^{2}}$. In order to guarantee existence of a unique $\hat{\theta}$ which solves (15), i.e., a unique $\hat{\theta}$ with $\hat{u}=\mathcal{G} \hat{\theta}$, we need injectivity of the linear map $\mathcal{G}$ or equivalently surjectivity of the adjoint map $\mathcal{G}^{*}$ (i.e., range $\mathcal{G}^{*}=\mathbb{R}^{p}$ ) (guaranteed, of course, if the functions $\left\{g_{i}\right\}$ are linearly independent in $L_{w}^{2}$ ). This is in turn equivalent to the fact that the linear map $\mathcal{G}^{*} \mathcal{G}: \mathbb{R}^{p} \rightarrow \mathbb{R}^{p}$ is 
bijective and in this case we obtain from (16) the unique solution

$$
\hat{\theta}\left(\theta_{0}\right)=\left(\mathcal{G}^{*} \mathcal{G}\right)^{-1} \mathcal{G}^{*} y\left(\cdot, \theta_{0}\right) .
$$

Without bijectivity, one often can obtain a unique solution with minimum norm (e.g., one typically minimizes over a compact set $\Theta_{A D}$ of parameters $\theta$ thereby guaranteeing that the range $\mathcal{R}(\mathcal{G})$ is closed in $L_{w}^{2}$-see [29]) by using the pseudoinverse $\mathcal{G}^{\dagger}$ in (17) in place of $\left(\mathcal{G}^{*} \mathcal{G}\right)^{-1} \mathcal{G}^{*}$. This also reveals that identifiability of the parameters $\theta$, which in the linear case is a global property, is equivalent to range $\mathcal{G}^{*}=\mathbb{R}^{p}$ or, equivalently, to invertibility of $\mathcal{G}^{*} \mathcal{G}$.

Using the representation $(\mathcal{G} \theta)(t)=G(t) \theta$ one can easily see that

$$
\mathcal{G}^{*} u=\left(\left\langle g_{1}, u\right\rangle_{L_{w}^{2}}, \ldots,\left\langle g_{p}, u\right\rangle_{L_{w}^{2}}\right)^{\top}, \quad u \in L_{w}^{2} .
$$

Taking $u=\mathcal{G} \theta=g_{1} \theta_{1}+\cdots+g_{p} \theta_{p}$ we obtain $\left\langle g_{i}, u\right\rangle_{L_{w}^{2}}=\left(\left\langle g_{i}, g_{1}\right\rangle_{L_{w}^{2}}, \ldots,\left\langle g_{i}, g_{p}\right\rangle_{L_{w}^{2}}\right) \theta$. From this we see that the 
matrix representation of the operator $\mathcal{G}^{*} \mathcal{G}$ is given by the $p \times p$-matrix

$$
\left(\left\langle g_{i}, g_{j}\right\rangle_{L_{w}^{2}}\right)_{i, j=1, \ldots, p}=\left(\int_{0}^{T} \frac{1}{\sigma^{2}(t)} g_{i}(t) g_{j}(t) d P(t)\right)_{i, j=1, \ldots, p} .
$$

This matrix is precisely the weighted Fisher information matrix for the linear case we are considering in this section (note that $\left.\nabla_{\theta} G(t) \theta=G(t)=\left(g_{1}(t), \ldots, g_{p}(t)\right), 0 \leq t \leq T, \theta \in \mathbb{R}^{p}\right)$.

From a statistical point of view, equation (17) relates the realizations $\hat{\theta}$ of the weighted least squares estimator $\hat{\Theta}$ to the measurements $y\left(\cdot, \theta_{0}\right)$ which is a realization of the stochastic process $Y\left(\cdot, \theta_{0}\right)$ given by

$$
Y\left(t, \theta_{0}\right)=G(t) \theta_{0}+\mathcal{E}(t), \quad 0 \leq t \leq T .
$$

An important question to be investigated is how the statistical properties of the estimator $\hat{\Theta}$ are related to the statistical properties 
of $Y$. The random variable equivalent of equation (17) is

$$
\hat{\Theta}\left(\theta_{0}\right)=\left(\mathcal{G}^{*} \mathcal{G}\right)^{-1} \mathcal{G}^{*} Y\left(\cdot, \theta_{0}\right) .
$$

From this equation we obtain for the expected value

$$
\begin{aligned}
E\left(\hat{\theta}\left(\theta_{0}\right)\right) & =E\left(\left(\mathcal{G}^{*} \mathcal{G}\right)^{-1} \mathcal{G}^{*} Y\left(\cdot, \theta_{0}\right)\right) \\
& =\left(\mathcal{G}^{*} \mathcal{G}\right)^{-1} E\left(\mathcal{G}^{*} Y\right) \\
& =\left(\mathcal{G}^{*} \mathcal{G}\right)^{-1} \mathcal{G}^{*} \mathcal{G} \theta_{0}=\theta_{0},
\end{aligned}
$$

where we have used $E\left(\mathcal{G}^{*} Y\right)=\mathcal{G}^{*} \mathcal{G} \theta_{0}$, which is a consequence of (19). Equation $(21)$ reveals that the estimator $\hat{\Theta}\left(\theta_{0}\right)$ is unbiased for 
any $\theta_{0} \in \mathbb{R}^{p}$. For the covariance matrix we obtain

$$
\begin{aligned}
\operatorname{Cov}\left(\hat{\Theta}\left(\theta_{0}\right)\right) & =\operatorname{Cov}\left(\left(\mathcal{G}^{*} \mathcal{G}\right)^{-1} \mathcal{G}^{*} Y\left(\cdot, \theta_{0}\right)\right) \\
& =\left(\mathcal{G}^{*} \mathcal{G}\right)^{-1} \operatorname{Cov}\left(\mathcal{G}^{*} Y\left(\cdot, \theta_{0}\right)\right)\left(\mathcal{G}^{*} \mathcal{G}\right)^{-1} \\
& =\left(\mathcal{G}^{*} \mathcal{G}\right)^{-1}\left(\mathcal{G}^{*} \mathcal{G}\right)\left(\mathcal{G}^{*} \mathcal{G}\right)^{-1}=\left(\mathcal{G}^{*} \mathcal{G}\right)^{-1}, \quad \theta_{0} \in \mathbb{R}^{p}
\end{aligned}
$$

In this equation we used the fact that

$$
\operatorname{Cov}\left(\mathcal{G}^{*} Y\left(\cdot, \theta_{0}\right)\right)=\mathcal{G}^{*} \mathcal{G} .
$$

Since the proof of this equation is not entirely trivial, we present it in some detail below. 
The $(i j)$-entry of the covariance matrix is

$$
\begin{aligned}
& \left(\operatorname{Cov}\left(G_{w}^{*} Y_{w}\right)\right)_{i j} \\
& =E\left(\int_{0}^{T} \frac{1}{\sigma^{2}(t)} g_{i}(t) \mathcal{E}(t) d P(t) \int_{0}^{T} \frac{1}{\sigma^{2}(s)} g_{j}(s) \mathcal{E}(s) d P(s)\right) .
\end{aligned}
$$

In order to establish (23) we must argue that

$$
\begin{array}{r}
E\left(\int_{0}^{T} \frac{1}{\sigma^{2}(t)} g_{i}(t) \mathcal{E}(t) d P(t)\right. \\
\left.\int_{0}^{T} \frac{1}{\sigma^{2}(s)} g_{j}(s) \mathcal{E}(s) d P(s)\right) \\
=\int_{0}^{T} \frac{1}{\sigma^{2}(t)} g_{i}(t) g_{j}(t) d P(t) .
\end{array}
$$


We consider two distinct cases of interest: (i) the case where the measure $P$ is absolutely continuous (in view of the weighings $\frac{1}{\sigma(t)}$ we can without loss of generality simple choose $P$ to be Lebesgue measure in this case) and (ii) $P$ is a discrete measure with a finite number of atoms. We first consider the argue for $P$ being Lebesgue measure.

From the independency assumption on $\mathcal{E}(t)$ we have that

$$
E(\mathcal{E}(t) \mathcal{E}(s))=\int_{\Omega} \mathcal{E}(t)(\omega) \mathcal{E}(s)(\omega) d \omega=\sigma(t) \sigma(s) \delta(t-s) .
$$

Multiplying this equation above by $g_{i}(t) / \sigma^{2}(t)$, integrating with respect to $t$ and changing the order of integration we obtain

$$
E\left(\int_{0}^{T} \frac{1}{\sigma^{2}(t)} g_{i}(t) \mathcal{E}(t) d t \mathcal{E}(s)\right)=g_{i}(s) .
$$


If we multiply now this equation with $g_{j}(s) / \sigma^{2}(s)$, and repeat the previous steps one more time (of course, now integrating with respect to $s$ ), we obtain

$$
E\left(\int_{0}^{T} \frac{1}{\sigma^{2}(t)} g_{i}(t) \mathcal{E}(t) d t \int_{0}^{T} \frac{1}{\sigma^{2}(s)} g_{j}(s) \mathcal{E}(s) d s\right)=\int_{0}^{T} \frac{1}{\sigma^{2}(s)} g_{i}(s) g_{j}(s) d s,
$$

which proves (24) in the case of Lebesgue measure. 
If instead of the Lebesgue measure we consider a discrete measure $P=\sum_{i=1}^{N} \delta_{t_{i}}$ with atoms at $t_{1}<t_{2}<\cdots<t_{n}$ in $[0, T]$, from the left side of (24), we have

$$
\begin{aligned}
E\left(\sum_{k=1}^{N} \frac{1}{\sigma^{2}\left(t_{k}\right)} g_{i}\left(t_{k}\right) \mathcal{E}\left(t_{k}\right)\right. & \left.\sum_{l=1}^{N} \frac{1}{\sigma^{2}\left(t_{l}\right)} g_{j}\left(t_{l}\right) \mathcal{E}\left(t_{l}\right)\right) \\
& =\sum_{k, l=1}^{N} \frac{1}{\sigma^{2}\left(t_{k}\right)} g_{i}\left(t_{k}\right) \frac{1}{\sigma^{2}\left(t_{l}\right)} g_{j}\left(t_{l}\right) E\left(\mathcal{E}\left(t_{k}\right) \mathcal{E}\left(t_{l}\right)\right) \\
& =\sum_{k, l=1}^{N} \frac{1}{\sigma^{2}\left(t_{k}\right)} g_{i}\left(t_{k}\right) \frac{1}{\sigma^{2}\left(t_{l}\right)} g_{j}\left(t_{l}\right) \sigma\left(t_{k}\right) \sigma\left(t_{l}\right) \delta_{k l} \\
& =\sum_{k=1}^{N} \frac{1}{\sigma^{2}\left(t_{k}\right)} g_{i}\left(t_{k}\right) g_{j}\left(t_{k}\right),
\end{aligned}
$$

which is (24) for the discrete case. 
The expected value and the covariance matrix of the parameter estimates $\hat{\theta}$ are important because they allow us to compute the confidence intervals for individual model parameters.

The expected value and the covariance matrix for the discrete time linear regression case is readily obtained from the continuous time case, if we observe that (see (18))

$$
\mathcal{G}^{*} \mathcal{G}=\left(\sum_{k=1}^{N} \frac{1}{\sigma^{2}\left(t_{k}\right)} g_{i}\left(t_{k}\right) g_{j}\left(t_{k}\right)\right)_{i, j=1, \ldots, p} .
$$

Moreover, the generalized weighted least square problem (14) becomes now a weighted least squares problem, i.e.,

$$
\hat{\theta}=\underset{\theta}{\operatorname{argmin}} \sum_{i=1}^{N} \frac{1}{\sigma^{2}\left(t_{i}\right)}\left(y\left(t_{i}\right)-(G \theta)\left(t_{i}\right)\right)^{2},
$$

whose solution $\hat{\theta}$ satisfies the corresponding discrete normal 
equations and has statistical properties which are the discrete equivalent of (21) and (22). Finally, we also note that for the linear regression case, the expected value (21) and the covariance matrix (22) are global with respect to $\theta_{0}$, that is they do not depend on the particular choice of the true parameter vector $\theta_{0}$.

We note that from the above discussions one may readily discern obvious connections between the needed well-conditioning of the discrete Fisher matrix (25), the requirements for $\mathcal{G}^{*} \mathcal{G}$ having full rank in the discussions for (17), the pseudoinverse theory for minimum norm solutions of least squares problems in Hilbert spaces presented in [29], and the asymptotic theory conditions involving nonsingularity of $\Omega$ in (??). 


\section{Nonlinear Regression - Distributed Measurements}

Assume now that we have a single output nonlinear regression model

$$
y(t)=f(t, \theta)+\varepsilon(t), \quad t \in[0, T],
$$

with distributed time measurements in the interval $[0, T]$ where $y(t)$ and $\varepsilon(t)$ are realizations of the corresponding statistical model processes $Y(t)$ and $\mathcal{E}(t)$. The process $\mathcal{E}(t)$ is a noisy process which describes the measurement error at time $t$, and is assumed to have the same properties as in the linear case. We shall apply the results from above to a linearized problem, thus obtaining only approximate statistical properties of the estimators in the nonlinear problem. The residual function in this case is $y(t)-f(t, \theta)$, and the weighted 
least squares problem becomes

$$
\hat{\theta}=\underset{\theta}{\operatorname{argmin}} \int_{0}^{T} \frac{1}{\sigma^{2}(t)}(y(t)-f(t, \theta))^{2} d P(t) .
$$

Again, $P$ is a measure (either continuous or discrete) which gives the distribution of the measurements in the interval $[0, T]$.

Similar to the analysis carried out for the linear case, in the following we will be interested in the statistical properties (i.e., mean and covariance matrix) of the estimator $\hat{\Theta}$. For the nonlinear regression problem we do not have the direct linear relationship (22) between the data and the estimated model parameters, and consequently we can no longer use the formulas (21) and (22). However, we will use a linearization approach to obtain approximations for the statistical properties of $\hat{\Theta}$. In a small neighborhood of $\theta_{0}$, we have the linear 
Taylor expansion

$$
f(t, \theta) \approx f\left(t, \theta_{0}\right)+\nabla_{\theta} f\left(t, \theta_{0}\right)\left(\theta-\theta_{0}\right) .
$$

Substituting this into the cost functional (26) we obtain

$$
\begin{aligned}
J(y, \theta) & =\int_{0}^{T} \frac{1}{\sigma^{2}(t)}(y(t)-f(t, \theta))^{2} d P(t) \\
& \approx \int_{0}^{T} \frac{1}{\sigma^{2}(t)}\left(y(t)-f\left(t, \theta_{0}\right)-\nabla_{\theta} f\left(t, \theta_{0}\right)\left(\theta-\theta_{0}\right)\right)^{2} d P(t) .
\end{aligned}
$$

We set $z(t)=y(t)-f\left(t, \theta_{0}\right)(=\varepsilon(t)), \beta=\theta-\theta_{0}$ and obtain

$$
J(y, \theta) \approx \tilde{J}(y, \beta):=\int_{0}^{T} \frac{1}{\sigma^{2}(t)}\left(z(t)-\nabla_{\theta} f\left(t, \theta_{0}\right) \beta\right)^{2} d P(t) .
$$

Let $\hat{\beta}$ be a minimizer of $\tilde{J}(y, \beta)$. Then $\tilde{\theta}=\hat{\beta}+\theta_{0}$ is (hopefully) a good approximation for $\hat{\theta}=\operatorname{argmin} J(y, \theta)$. The big advantage of using the linearization of $f$ around $\theta_{0}$ is that it leads to a linear least 
squares problem for $\beta$. The solution $\hat{\beta}$ is given by the normal equations (16),

$$
\hat{\beta}=\left(G_{w}^{*} G_{w}\right)^{-1} G_{w}^{*} z,
$$

where the operator $G_{w}$ now is given by

$$
G_{w}=\nabla_{\theta} f_{w}\left(\cdot, \theta_{0}\right),
$$

i.e., we have $g_{j}(t)=\left(\partial f / \partial \theta_{j}\right)\left(t, \theta_{0}\right), j=1, \ldots, p$. As in Section 26 we tacitly also assume here that $\operatorname{rank} G=p$, which implies that the $p \times p$-matrix $G_{w}^{*} G_{w}$ is invertible. From (27) we obtain

$$
\hat{\theta}-\theta_{0} \approx\left(G_{w}^{*} G_{w}\right)^{-1} G_{w}^{*} z .
$$

The non-singular matrix $G_{w}^{*} G_{w}$ is the $p \times p$ weighted Fisher information matrix

$$
F\left(T, \theta_{0}\right)=\int_{0}^{T} \frac{1}{\sigma^{2}(t)} \nabla_{\theta}^{\top} f\left(t, \theta_{0}\right) \nabla_{\theta} f\left(t, \theta_{0}\right) d P(t)
$$


Applying the results obtained above for linear regression models with distributed measurements, we have the approximate relationships

$$
E(\hat{\Theta})=\theta_{0},
$$

and

$$
\operatorname{Cov}(\hat{\Theta})=F\left(T, \theta_{0}\right)^{-1} .
$$

Thus we have

$$
\hat{\Theta} \sim \mathcal{N}_{p}\left(\theta_{0}, F\left(T, \theta_{0}\right)^{-1}\right) .
$$




\section{Traditional and Generalized Sensitivity Functions}

\section{Traditional Sensitivity Functions}

The traditional sensitivity functions are frequently used in simulation studies (i.e., when we investigate forward problems) where one wants to assess the degree of sensitivity of a model output with respect to various parameters on which it depends, and to identify the parameters to which the model is most/least sensitive. We consider the output model (2). To quantify the variation in the output variable $\eta(t)$ with respect to changes in the $k$-th component $\theta_{k}$ of the parameter vector $\theta$ we are led to consider the first order sensitivity functions, also called the traditional sensitivity functions (TSF), 
defined naturally in terms of the partial derivatives

$$
s_{k}(t, \theta)=\frac{\partial \eta}{\partial \theta_{k}}(t, \theta) \in \mathbb{R}^{M}, \quad k=1, \ldots, p,
$$

which assumes smoothness of $f$ with respect $\theta$ (compare $[2,16,19,22,27],[28$, pp. $7-9]$, [33]). If the output model (2) originates from the dynamical system (1) and we assume sufficient regularity of the functions $\mathcal{F}, x_{0}(\cdot), h$, then the $M \times p$ matrix

$$
s(t, \theta)=\left(s_{1}(t, \theta), \ldots, s_{p}(t, \theta)\right)=\left(\begin{array}{c}
\nabla_{\theta} \eta_{1}(t, \theta) \\
\vdots \\
\nabla_{\theta} \eta_{M}(t, \theta)
\end{array}\right)
$$

is given by

$$
s(t, \theta)=\frac{\partial h}{\partial x}(t, x(t, \theta), \theta) \frac{\partial x}{\partial \theta}(t, \theta)+\frac{\partial h}{\partial \theta}(t, x(t, \theta), \theta), \quad 0 \leq t \leq T,
$$


where $\partial h / \partial x=\left(\partial h_{i} / \partial x_{j}\right)_{i=1, \ldots, M, j=1, \ldots, N}$,

$\partial x / \partial \theta=\left(\partial x_{i} / \partial \theta_{k}\right)_{i=1, \ldots, N, k=1, \ldots, p}$ and

$\partial h / \partial \theta=\left(\partial h_{i} / \partial \theta_{k}\right)_{i=1, \ldots, M, k=1, \ldots, p}$.

It is well known that the matrix $X(t, \theta):=(\partial x / \partial \theta)(t, \theta) \in \mathbb{R}^{N \times p}$ as a function of $t$ satisfies the linear ODE-system (called the sensitivity equations)

$$
\begin{aligned}
\dot{X}(t, \theta) & =\mathcal{F}_{x}(t, x(t, \theta), \theta) X(t, \theta)+\mathcal{F}_{\theta}(t, x(t, \theta), \theta), \\
X(0, \theta) & =\frac{\partial x_{0}}{\partial \theta}(\theta),
\end{aligned}
$$

where $x(t, \theta)$ is the solution of the initial value problem in (1). The matrices $\mathcal{F}_{x} \in \mathbb{R}^{N \times N}$ and $\mathcal{F}_{\theta} \in \mathbb{R}^{N \times p}$ are defined analogous to the matrices $\partial h / \partial x$, etc., above. Equation (30) is very useful in practice, since it provides in conjunction with system (1) a fast and efficient way to compute the sensitivity matrix $s$ numerically for general 
systems of the form (1).

The sensitivity functions (29) are in fact simply the derivatives of the components of the output $\eta$ with respect to the parameters. As long as one is only interested in the sensitivity of the output solely with respect to individual parameters (as in this paper), it is sufficient to use the functions given by (29). However, if we want to compare the sensitivity of outputs with respect to different parameters, then the derivatives (29) can be misleading. Instead of the derivatives (29) one should use the relative sensitivity functions $\sigma_{k}$ defined by

$$
\begin{array}{r}
\sigma_{k}(t, \theta)=\lim _{\Delta \rightarrow 0} \frac{\left(\eta\left(t, \theta+\Delta e_{k}\right)-\eta(t, \theta)\right) / \eta(t, \theta)}{\Delta / \theta_{k}} \\
=\frac{\theta_{k}}{\eta(t, \theta)} \frac{\partial \eta}{\partial \theta_{k}}(t, \theta)=\frac{\theta_{k}}{\eta(t, \theta)} s_{k}(t, \theta) .
\end{array}
$$

A further advantage of the functions $\sigma_{k}$ is that they are dimensionless. 
Although the first order sensitivity functions (29) are among the most commonly used tools in simulation studies, sometimes it is useful to consider also the second order sensitivity functions defined by

$$
s_{k, m}(t, \theta)=\frac{\partial^{2} \eta}{\partial \theta_{k} \partial \theta_{m}}(t, \theta), \quad k, m=1, \ldots, p .
$$

The function $s_{k, m}$ is a measure for the variation of the sensitivity $s_{m}$ when the parameter $\theta_{k}$ changes or, vice versa, for the variation of $s_{k}$ when $\theta_{m}$ changes. 
(a) Logistic curve

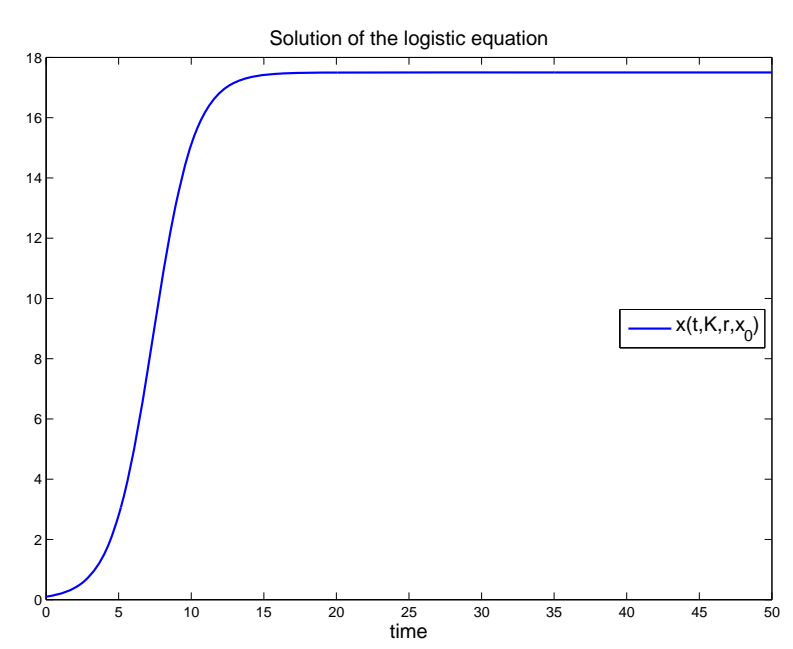

(b) TSF

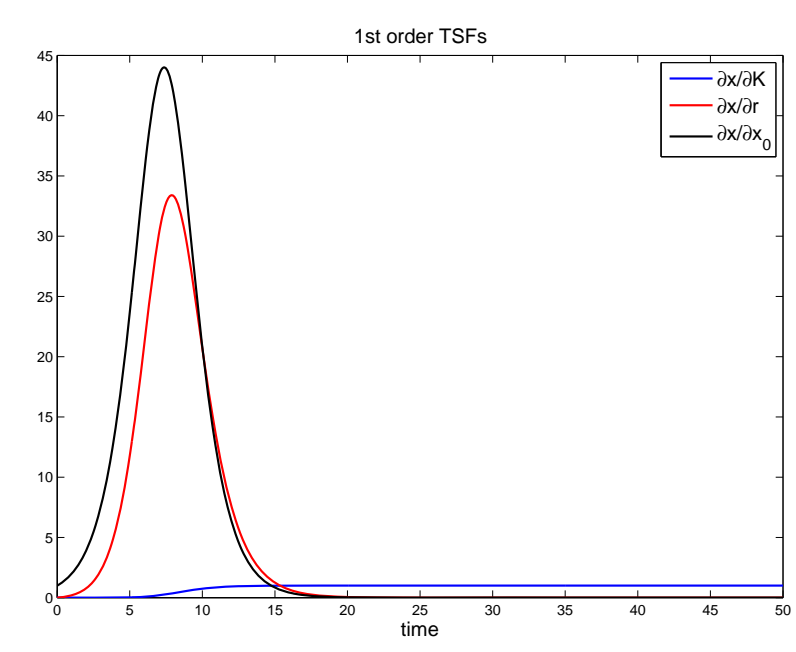

Figure 1: (a) Logistic curve; (b) TSF for logistic model 
(c) $2^{\text {nd }}$ order TSF, diags

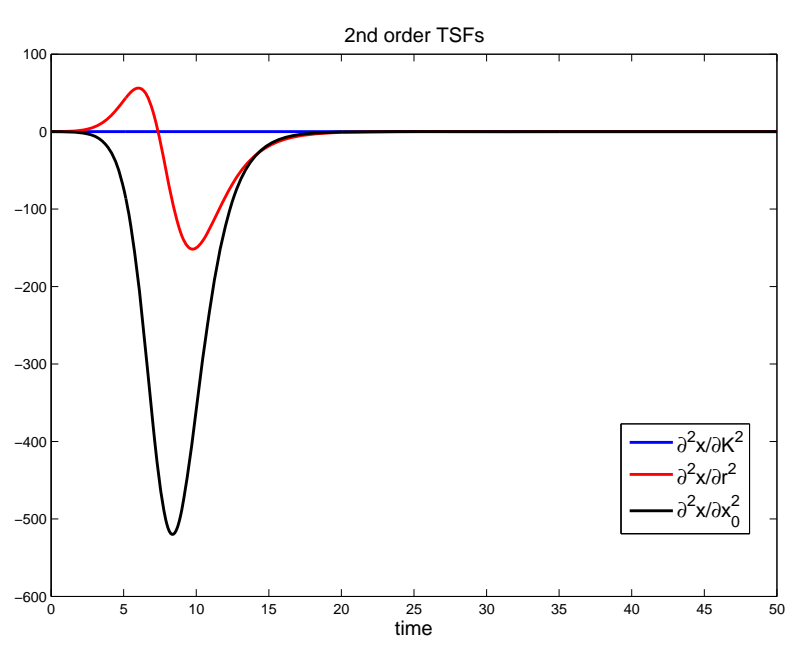

(d) $2^{\text {nd }}$ order TSF, off-diags

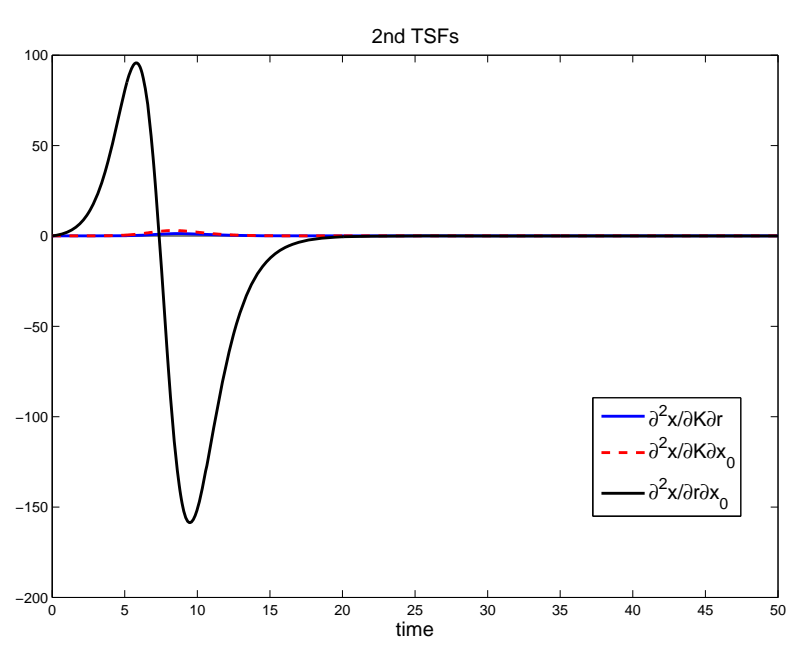

Figure 2: (c) Second order TSF for logistic model, diagonal entries; (d) Second order TSF for logistic model, off-diagonal entries 
(e) $1^{\text {st }} \& 2^{\text {nd }}$ order TSF wrt $r$

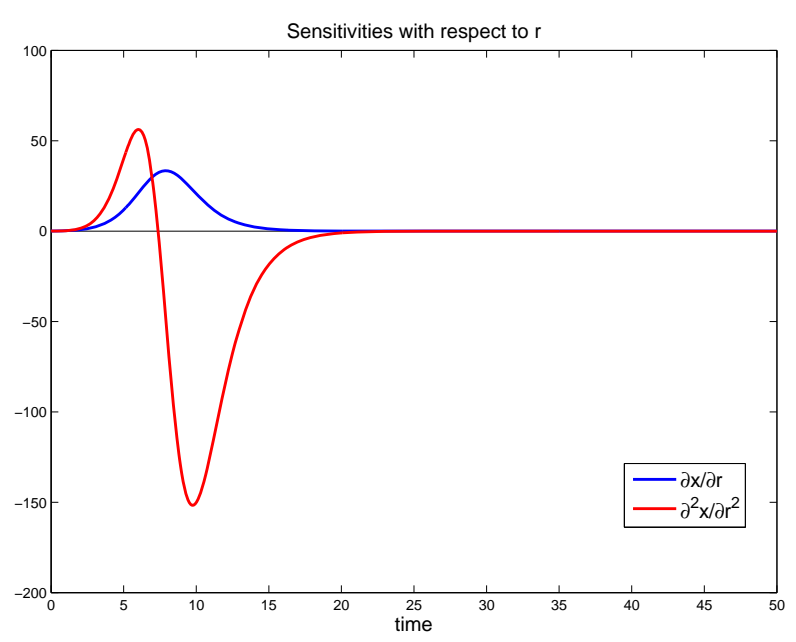

(f) $1^{\text {st }} \& 2^{\text {nd }}$ order TSF wrt $x_{0}$

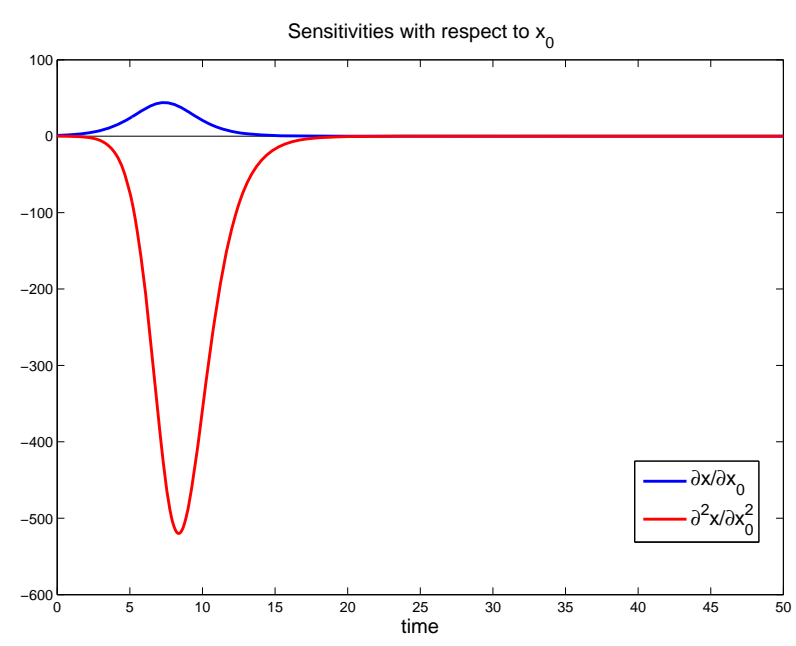

Figure 3: (e) First and second order TSF with respect to $r$; (f) First and second order TSF with respect to $x_{0}$. All the figures above were plotted for the true parameter vector $\theta_{0}=(17.5,0.7,0.1)$. 
It is important to note that the (first and second order) sensitivity functions depend on both $t$ and $\theta$, and it is this double dependence that makes them suitable as optimal design tools. The TSF characterize the rate of change of the output of a model (as a function of time) with respect to a parameter, but being derivatives with respect to the parameters this characterization is only local with respect to the parameters. For example, if the sensitivity $s_{k}$ is close to zero on a time subinterval $[\tilde{t}-\delta, \tilde{t}+\delta]$ and for $\theta$ in a neighborhood of the true value $\theta_{0}$, then the output given by (2) is insensitive to the parameter $\theta_{k}$ on that particular time subinterval and in a neighborhood of $\theta_{0}$. The same function $s_{k}$ can take large values on a different time subinterval or in a neighborhood of a different nominal parameter vector $\tilde{\theta}_{0}$, indicating that the output variable $\eta$ is very sensitive to the parameter $\theta_{k}$ on the latter time subinterval in a neighborhood of $\tilde{\theta}_{0}$. 
Now turn to derive briefly an expression involving a second order sensitivity-useful in subsequent discussions (see discussions of sets $S_{[5,10]}^{*}$ below)-consider the Taylor expansion of the regression function $f(t, \theta)$ in $(5)$ around the true value $\theta_{0}$, i.e., $f(t, \theta)=f\left(t, \theta_{0}\right)+\nabla_{\theta} f\left(t, \theta_{0}\right)\left(\theta-\theta_{0}\right)+\frac{1}{2}\left(\theta-\theta_{0}\right)^{\top} \nabla_{\theta \theta}^{2} f\left(t, \theta_{0}\right)\left(\theta-\theta_{0}\right)+\ldots$. In a small neighborhood of $\theta_{0}$, second order Taylor polynomial is a good approximation of $f$-substitute in (9) and minimize instead the approximate cost functional $\tilde{J}(y, \theta)$, given by

$$
\begin{aligned}
\tilde{J}(y, \theta)=\int_{0}^{T} \frac{1}{\sigma^{2}(t)}(\varepsilon(t)- & \nabla_{\theta} f\left(t, \theta_{0}\right)\left(\theta-\theta_{0}\right) \\
& \left.-\frac{1}{2}\left(\theta-\theta_{0}\right)^{\top} \nabla_{\theta \theta}^{2} f\left(t, \theta_{0}\right)\left(\theta-\theta_{0}\right)\right)^{2} d P(t) .
\end{aligned}
$$


In a neighborhood of $\theta_{0}, J(y, \theta) \approx \tilde{J}(y, \theta)$ and the value $\tilde{\theta}$ which minimizes $\tilde{J}(y, \theta)$ is a good approximation for the estimate $\hat{\theta}$ given by (10). The optimality condition at $\tilde{\theta}$ is simply $\nabla_{\theta} \tilde{J}(y, \tilde{\theta})=0$, or equivalently

$$
\begin{aligned}
2 \int_{0}^{T} \frac{1}{\sigma^{2}(t)}(\varepsilon(t) & \left.-\nabla_{\theta} f\left(t, \theta_{0}\right)\left(\tilde{\theta}-\theta_{0}\right)-\frac{1}{2}\left(\tilde{\theta}-\theta_{0}\right)^{\top} \nabla_{\theta \theta}^{2} f\left(t, \theta_{0}\right)\left(\tilde{\theta}-\theta_{0}\right)\right) \\
& \times\left(\nabla_{\theta} f\left(t, \theta_{0}\right)+\left(\tilde{\theta}-\theta_{0}\right)^{\top} \nabla_{\theta \theta}^{2} f\left(t, \theta_{0}\right)\right)^{\top} d P(t)=0_{p \times 1} .
\end{aligned}
$$

If we keep only the first order terms in $\tilde{\theta}-\theta_{0}$ in the equation above, we obtain

$$
A\left(\tilde{\theta}-\theta_{0}\right) \approx \int_{0}^{T} \frac{\varepsilon(t)}{\sigma^{2}(t)} \nabla_{\theta}^{\top} f\left(t, \theta_{0}\right) d P(t),
$$

where the matrix $A$ is given by

$$
A=\int_{0}^{T} \frac{1}{\sigma^{2}(t)}\left(\nabla_{\theta}^{\top} f\left(t, \theta_{0}\right) \nabla_{\theta} f\left(t, \theta_{0}\right)-\varepsilon(t) \nabla_{\theta \theta}^{2} f\left(t, \theta_{0}\right)\right) d P(t) .
$$


In Figures 1 and 2 we plotted logistic growth curve along with first and second order traditional sensitivity functions. The magnitudes of second order sensitivity functions are much larger than those of first order sensitivity functions. Moreover, their support is slightly wider, as can be seen in Figure 3(e) and (f). These facts support our suggestions that second order sensitivity functions may play an important role, and they should be used in conjunction with first order sensitivity functions in parameter estimation problems.

Conclude: intuitive comment that if we want to improve accuracy of a parameter $\theta_{k}$, we should take extra measurements at time points where the first order sensitivity functions (TSF) are large and the second order sensitivities are small (or where relative magnitudes considerably favor the TSF)-Based on experience, advocate use of first and second order sensitivity functions as optimal design tools. 


\section{Sensitivity of Estimates with Respect to Model Parameters and Generalized Sensitivity Functions}

As we have already noted, the sensitivity functions introduced in the previous subsection are mainly used in simulation studies, where one wants to ascertain the change in model output with respect to changes in parameters. However, as we suggested they can be used also as optimal design tools. In this subsection we build mathematical tools which directly quantify the sensitivity of the parameter estimates $\hat{\theta}$ with respect to the model parameters $\theta$. As with the traditional sensitivity functions, our approach will be motivated and carried out from an optimal design perspective. Our starting point will again be the optimality condition (7) for the least squares problem with general measurements procedures. Our discussions here are based directly on the ideas of Thomaseth and Cobelli as discussed in 


\section{$[12,35]$.}

Under reasonable assumptions, the cost functional $J$ in $(7)$ is differentiable with respect to $\theta$. Therefore the estimate $\hat{\theta}\left(\theta_{0}\right)$ corresponding to a given $y\left(\cdot, \theta_{0}\right)$ satisfies the optimality condition

$$
\nabla_{\theta} J\left(y\left(\cdot, \theta_{0}\right), \hat{\theta}\right)=0,
$$

or equivalently

$$
\int_{0}^{T} \frac{1}{\sigma^{2}(s)}\left(y\left(s, \theta_{0}\right)-f(s, \hat{\theta})\right) \nabla_{\theta} f(s, \hat{\theta}) d P(s)=0 .
$$


Consider nominal parameter vectors $\theta$ in neighborhood $\mathcal{U}$ of $\theta_{0}$ with corresponding estimates $\hat{\theta}=\hat{\theta}(\theta), \theta \in \mathcal{U}$-condition (34) holds for all $\theta \in \mathcal{U}$, i.e.,

$g(\theta):=\int_{0}^{T} \frac{1}{\sigma^{2}(s)}(y(s, \theta)-f(s, \hat{\theta}(\theta))) \nabla_{\theta} f(s, \hat{\theta}(\theta)) d P(s)=0, \quad \theta \in \mathcal{U}$.

Implies that derivative $D g$ of $g$ with respect to $\theta$ is zero in $\mathcal{U}$ or

$$
D g(\theta)=0, \quad \theta \in \mathcal{U} \text {. }
$$

Since $\theta \mapsto g(\theta)$ defines a mapping $\mathcal{U} \rightarrow \mathbb{R}^{1, p} \cong \mathcal{L}\left(\mathbb{R}^{p}, \mathbb{R}\right)$, we see that $D g(\theta) \in \mathcal{L}\left(\mathbb{R}^{p}, \mathcal{L}\left(\mathbb{R}^{p}, \mathbb{R}\right)\right) \cong \mathcal{L}\left(\mathbb{R}^{p} \times \mathbb{R}^{p}, \mathbb{R}\right)$, i.e., $D g(\theta)$ is a bilinear functional on $\mathbb{R}^{p}$ and thus can be represented by a $p \times p$ matrix (see, for instance, [18, Section VIII.12]). 
In order to obtain $D g(\theta)$ we expand $g(\theta+\delta), \delta \in \mathbb{R}^{p}$, as

$$
g(\theta+\delta)=g(\theta)+D g(\theta) \delta+\text { higher order terms, }
$$

and obtain after some straight forward computations

$$
\begin{aligned}
0=D g(\theta)= & \nabla_{\theta \theta}^{2} J(y(\cdot, \theta), \hat{\theta}(\theta)) \frac{\partial \hat{\theta}}{\partial \theta}(\theta)+\nabla_{\theta y}^{2} J(y(\cdot, \theta), \hat{\theta}(\theta)) \nabla_{\theta} y(\cdot, \theta) \\
= & \left(\int_{0}^{T} \frac{1}{\sigma^{2}(s)}(y(s, \theta)-f(s, \hat{\theta}(\theta))) \nabla_{\theta \theta}^{2} f(s, \hat{\theta}(\theta)) d P(s)\right. \\
& \left.\quad-\int_{0}^{T} \frac{1}{\sigma^{2}(s)} \nabla_{\theta}^{\top} f(s, \hat{\theta}(\theta)) \nabla_{\theta} f(s, \hat{\theta}(\theta)) d P(s)\right) \frac{\partial \hat{\theta}}{\partial \theta}(\theta) \\
& +\int_{0}^{T} \frac{1}{\sigma^{2}(s)} \nabla_{\theta}^{\top} f(s, \hat{\theta}(\theta)) \nabla_{\theta} y(s, \theta) d P(s), \quad \theta \in \mathcal{U} .
\end{aligned}
$$


From this equation we can obtain the sensitivity matrix $\partial \hat{\theta} / \partial \theta$ provided $\nabla_{\theta \theta}^{2} J(y(\cdot, \theta), \hat{\theta}(\theta))$ is nonsingular. We are interested in how this sensitivity matrix changes if we progress with our measurement procedure in time. Thus we may intuitively view this methodology as one which attempts to measure how observations (data) contribute longitudinally to our ability to estimate $\hat{\theta}$ through the sensitivity of $\hat{\theta}$ with respect to a particular component $\theta_{k}$. That is, we are describing a sensitivity of the estimated parameters with respect to the observations or data concept. In view of this goal we assume that, for some fixed $t \in[0, T]$, a variation of the nominal parameter vector $\theta$ only causes a change of the measurements taken during the time interval $[0, t]$, whereas measurements taken during $(t, T]$ remain fixed to their original values. 
Therefore we have (using also that $\varepsilon(t)$ is not dependent on $\theta$ )

$$
\nabla_{\theta} y(s, \theta)= \begin{cases}\nabla_{\theta} f(s, \theta) & \text { for } 0 \leq s \leq t \\ 0 & \text { for } t<s \leq T\end{cases}
$$

which implies

$$
\begin{aligned}
& \nabla_{\theta y}^{2} J(y(\cdot, \theta), \hat{\theta}(\theta)) \nabla_{\theta} y(\cdot, \theta) \\
& \quad=\int_{0}^{t} \frac{1}{\sigma^{2}(s)} \nabla_{\theta}^{\top} f(s, \hat{\theta}(\theta)) \nabla_{\theta} f(s, \theta) d P(s), \quad \theta \in \mathcal{U} .
\end{aligned}
$$


Equation (35) relates the sensitivity of the estimates $\hat{\theta}$ with respect to $\theta$, to the corresponding sensitivities of the output. The entries of the sensitivity matrix $\partial \hat{\theta} / \partial \theta$ are the quantities of interest. They depend on the model parameters $\theta$, on $t \in[0, T]$ and on the final time $T$. Naturally, equation (35) is our starting point for investigating the behavior of sensitivities $\partial \hat{\theta} / \partial \theta=(\partial \hat{\theta} / \partial \theta)(t)$ as functions of $t$ and $\theta$. However, there is an important disadvantage associated with this equation, namely the fact that it is realization-dependent. The sensitivities $\partial \hat{\theta} / \partial \theta$ are given in terms of $\hat{\theta}(\theta)$ and $y(\cdot, \theta)$, which are realizations of the corresponding least squares estimator $\hat{\Theta}(\theta)$ and of the random variable $Y(\cdot, \theta)$ which governs the measurement process on the time interval $[0, T]$. 
Our ultimate goal is to develop mathematical tools that indicate regions of high information content with respect to a parameter where the tools are not realization-dependent. Therefore, we next consider equation (35) formulated in terms of the random variables $\hat{\Theta}$ and $Y$, i.e.,

$$
\left(\nabla_{\theta \theta}^{2} J(Y(\cdot, \theta), \hat{\Theta}(\theta))\right) \frac{\partial \hat{\Theta}}{\partial \theta}(\theta)=-\nabla_{\theta y}^{2} J(Y(\cdot, \theta), \hat{\Theta}(\theta)) \frac{\partial Y}{\partial \theta}(\cdot, \theta),
$$

and instead of investigating the behavior of the sensitivities $\partial \hat{\theta} / \partial \theta$ for a particular realization, we will be interested instead in the behavior of the expected value

$$
E\left(\frac{\partial \hat{\Theta}}{\partial \theta}(\theta)(t)\right), \quad 0 \leq t \leq T, \theta \in \mathcal{U},
$$

as a function of $t$ and $\theta$. 
From asymptotic statistical theory, we know that the estimator $\hat{\Theta}$ is unbiased, i.e., $E(\hat{\Theta}(\theta))=\theta$ in a neighborhood $\mathcal{U}$ of $\theta_{0}$. Using this, we can argue that $E\left(\nabla_{\theta} f(t, \hat{\Theta}(\theta))\right) \approx \nabla_{\theta} f(t, \theta)$, and using additional technical assumptions in equation (35) (expected value commuting with the time integral, expected value of products approximately equal to the product of expected values), we obtain that the expected value of $\nabla_{\theta \theta}^{2} J$ is approximately given by

$$
\begin{aligned}
& E\left(\nabla_{\theta \theta}^{2} J(Y(\cdot, \theta), \hat{\Theta}(\theta)) \approx\right. \\
& \int_{0}^{T} \frac{1}{\sigma^{2}(s)} \nabla_{\theta}^{\top} f(s, \theta) \nabla_{\theta} f(s, \theta) d P(s)=: F(T, \theta),
\end{aligned}
$$

which we assume to be nonsingular. We note that the $p \times p$ matrix $F(T, \theta)$ on the right side of (37), is simply the Fisher information matrix (FIM) for our problem with respect to general measurement procedure determined by $P$ in the interval $[0, T]$. As one can see from 
its definition, the Fisher information matrix depends on the mathematical model $f$, the measure $P$ and the final time $T$. An interesting question to address is if the evolution of the condition number of $F$ as a function of $T$ can be used to choose an appropriate stopping time for our experiments. We will carry out this analysis later. 
Under similar conditions which led to equation (37) we obtain

$$
\begin{aligned}
& E\left(\nabla_{\theta y}^{2} J(Y(\cdot, \theta), \hat{\Theta}(\theta)) \frac{\partial Y}{\partial \theta}(\cdot, \theta)\right) \approx \\
& \int_{0}^{t} \frac{1}{\sigma^{2}(s)} \nabla_{\theta}^{\top} f(s, \theta) \nabla_{\theta} f(s, \theta) d P(s)=F(t, \theta) .
\end{aligned}
$$

It is important to note that if $r=r(\theta)$ is a linear function then $E(r(\theta))=r(E(\theta))$, which consequently holds only up to a first-order approximation when $r$ is nonlinear; in general we only have $E(r(\theta)) \approx r(E(\theta))$. 
Next we assume that $\left(\nabla_{\theta \theta}^{2} J(Y, \hat{\Theta})\right)^{-1}$ and $\nabla_{\theta y}^{2} J(Y, \hat{\Theta})(\partial Y / \partial \theta)$ are independent $p \times p$ random variables, which implies that

$$
\begin{aligned}
E\left(\left(\nabla_{\theta \theta}^{2} J(Y, \hat{\Theta})\right)^{-1}\right. & \left.\nabla_{\theta y}^{2} J(Y, \hat{\Theta}) \frac{\partial Y}{\partial \theta}\right) \\
& =E\left(\left(\nabla_{\theta \theta}^{2} J(Y, \hat{\Theta})\right)^{-1}\right) E\left(\nabla_{\theta y}^{2} J(Y, \hat{\Theta}) \frac{\partial Y}{\partial \theta}\right) .
\end{aligned}
$$


If we now take expected values in equation (35), and use the equation above along with (37) and (38), we obtain

$$
E\left(\frac{\partial \hat{\Theta}}{\partial \theta}(\theta)\right)(t) \approx F^{-1}(T, \theta) F(t, \theta)=: G(T, t, \theta), \quad 0 \leq t \leq T, \theta \in \mathcal{U} .
$$

Equation (40) is realization-independent and gives the evolution of the expected value $E(\partial \hat{\Theta} / \partial \theta)$ as a function of $t$ and $\theta$ when $t \in[0, T]$ and $\theta$ is in a neighborhood of $\theta_{0}$. For $t=T$ we see that

$$
E\left(\frac{\partial \hat{\Theta}}{\partial \theta}(\theta)\right)(T)=I_{p \times p}
$$

which reflects the assumption that the estimator $\hat{\Theta}$ is unbiased (i.e., the variation in parameter estimates is equal to the variation in the true model parameters [35]). 
We call the diagonal elements of the matrix $F^{-1}(T, \theta) F(t, \theta)$ the generalized sensitivity functions (GSF) with respect to the parameters $\theta$,

$$
\begin{array}{r}
g s(t, \theta)=\operatorname{diag}(G(T, t, \theta)) \\
=\operatorname{diag}\left(\int_{0}^{t} F(T, \theta)^{-1} \frac{1}{\sigma^{2}(s)} \nabla_{\theta}^{\top} f\left(s, \theta_{0}\right) \nabla_{\theta} f\left(s, \theta_{0}\right) d P(s)\right), \\
t \in[0, T] .
\end{array}
$$


If $P$ is the discrete measure (8) then we obtain precisely the generalized sensitivity functions as introduced by Thomaseth and Cobelli in [35]. Of course, the vector $g s(t, \theta)$ can also be written as $g s(t, \theta)=\int_{0}^{t}\left(F(T, \theta)^{-1} \frac{1}{\sigma^{2}(s)} \nabla_{\theta}^{\top} f\left(s, \theta_{0}\right)\right) \bullet \nabla_{\theta} f\left(s, \theta_{0}\right) d P(s), \quad t \in[0, T]$,

where the symbol "•" denotes the element-by-element multiplication of two vectors. From (40) we see that

$$
g s(t, \theta) \approx \operatorname{diag}\left(E\left(\frac{\partial \hat{\Theta}}{\partial \theta}(T, \theta)\right)(t)\right) .
$$

Hence the GSF approximate the diagonal elements of the expected value matrix of the sensitivities of the estimator $\hat{\Theta}$ with respect to $\theta$ in (40), regarded as time-dependent functions on $[0, T]$. 
Like discrete counterparts introduced by Thomaseth and Cobelli, GSF (41) illustrate how information content wrt the parameters to be estimated is distributed throughout experiment. By definition, GSF are cumulative functions- sometimes misleading-exhibit false regions of high information content (the so called "forced-to-one" artifact [3, 8]; see also [26], where it is shown that if $G(T, \cdot, \theta)$ is close to a linear functions for $t$ in some interval $\left[T_{1}, T\right], T_{1}<T$, then the parameter estimation problem with measurements taken only in $\left[T_{1}, T\right]$ is ill-posed) To avoid potential misunderstanding caused by these artifacts, also consider first time derivative of the GSF, which in case that $P$ is the Lebesgue measure on $[0, T]$ is given by

$$
\frac{\partial}{\partial t} g s(t, \theta)=\left(F(T, \theta)^{-1} \frac{1}{\sigma^{2}(t)} \nabla_{\theta}^{\top} f(t, \theta)\right) \bullet \nabla_{\theta} f(t, \theta),
$$

which is related to the incremental generalized sensitivities introduced in [35]. 
(a) GSFs for $T=10$

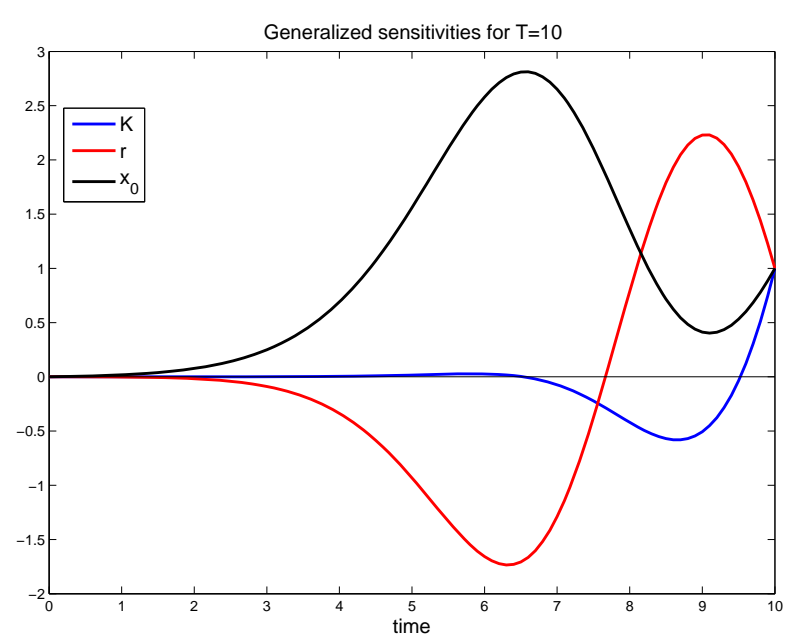

(c) GSFs for $T=50$

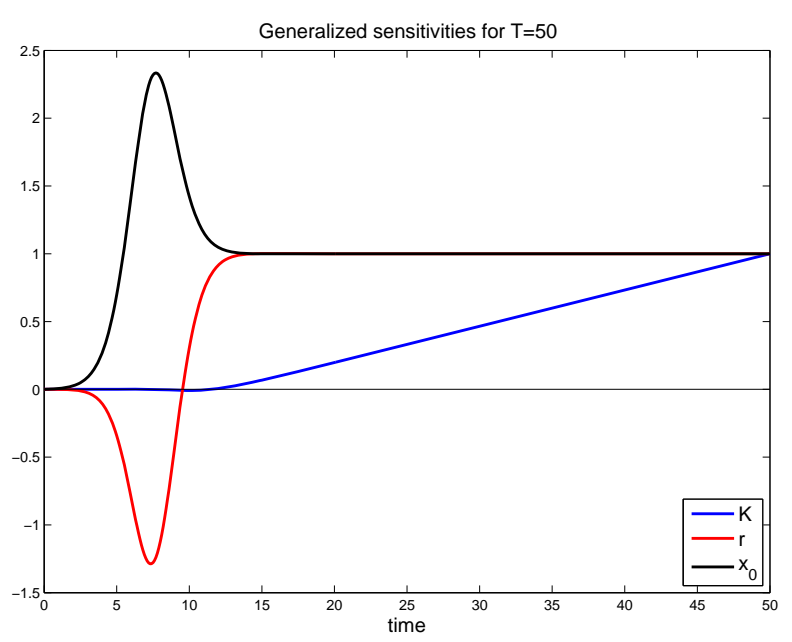

(b) GSFs for $T=20$

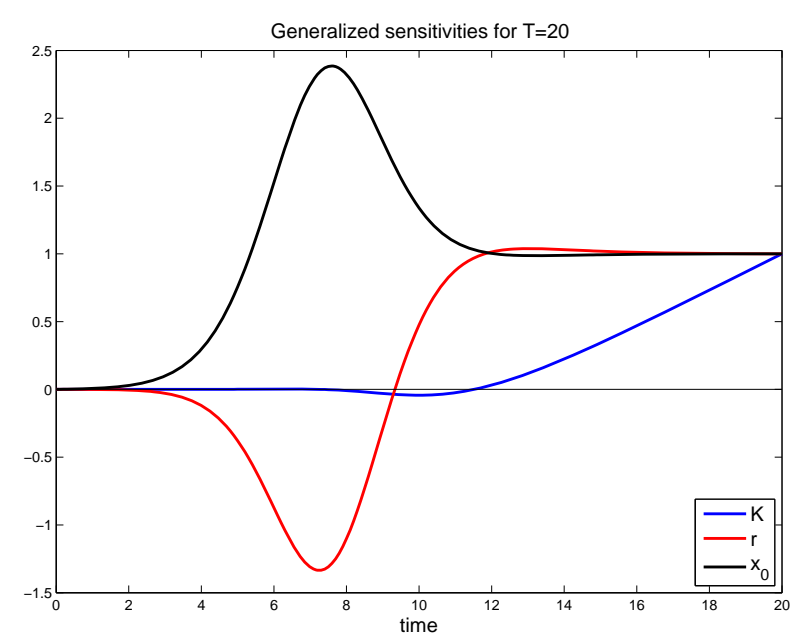

(d) time derivatives GSFs for $T=50$

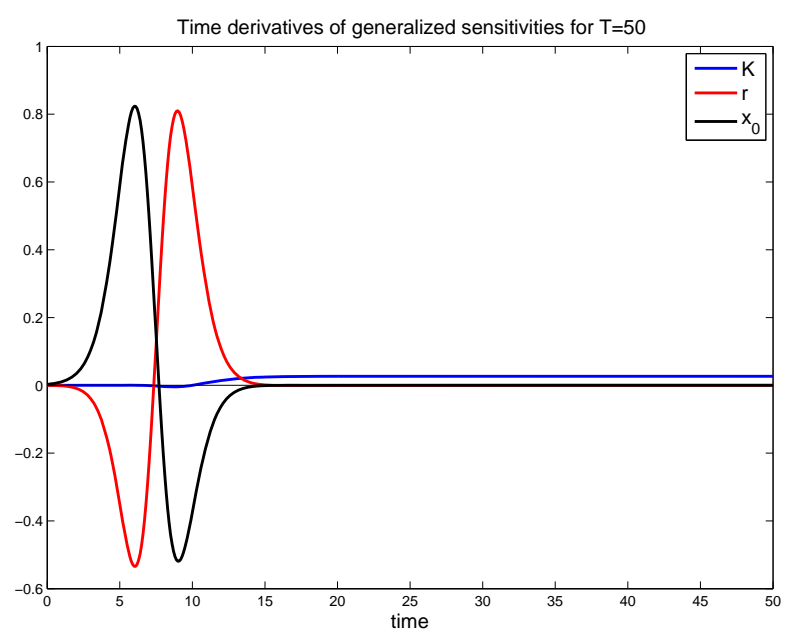


Remark 0.1. a) If $P=P_{d}$ as given by (8) then we obtain

$$
F_{d}(t, \theta)= \begin{cases}0 & \text { for } 0 \leq t<t_{1} \\ \sum_{i=1}^{k} \frac{1}{\sigma^{2}\left(t_{i}\right)} \nabla_{\theta}^{\top} f\left(t_{i}, \theta\right) \nabla_{\theta} f\left(t_{i}, \theta\right) & \text { for } t_{k} \leq t<t_{k+1}, k=1, \ldots, n \\ \sum_{i=1}^{n} \frac{1}{\sigma^{2}\left(t_{i}\right)} \nabla_{\theta}^{\top} f\left(t_{i}, \theta\right) \nabla_{\theta} f\left(t_{i}, \theta\right) & \text { for } t_{n} \leq t \leq T\end{cases}
$$

In this case the generalized sensitivity functions $g s(t, \theta)$ are piecewise constant functions with $g s(0, \theta)=(0, \ldots, 0)$, $g s(T, \theta)=(1, \ldots, 1)$ and jumps of size

$$
\operatorname{diag}\left(\left(\sum_{i=1}^{n} \frac{1}{\sigma^{2}\left(t_{i}\right)} \nabla_{\theta}^{\top} f\left(t_{i}, \theta\right) \nabla_{\theta} f\left(t_{i}, \theta\right)\right)^{-1} \nabla_{\theta}^{\top} f\left(t_{i}, \theta\right) \nabla_{\theta} f\left(t_{i}, \theta\right)\right)
$$

at $t_{i}, i=1, \ldots, n$. 
b) Let $P$ be the Lebesgue measure and let $F$ be the Fisher information matrix in this case (as given by (38)). Assume that $0 \leq t_{1}^{(n)}<\cdots<t_{N(n)}^{(n)}=T, n=1,2, \ldots$, is a sequence of meshes in $[0, T]$ with $\max _{i=1, \ldots, N(n)}\left(t_{i}^{(n)}-t_{i-1}^{(n)}\right) \rightarrow 0$ as $n \rightarrow \infty$ (here we set $t_{0}^{(n)}=0$ for all $\left.n\right)$. Let $F_{n}$ denote the Fisher information matrix corresponding to the discrete measure $P_{n}=\sum_{i=1}^{N(n)}\left(t_{i}^{(n)}-t_{i-1}^{(n)}\right) \delta_{t_{i}^{(n)}}$. Then it is easy to see that

$$
\lim _{n \rightarrow \infty} F_{n}(t, \theta)=F(t, \theta), \quad 0 \leq t \leq T, \theta \in \mathcal{U} .
$$

If the meshes are uniform with mesh size $t_{i}^{(n)}-t_{i-1}^{(n)}=T / N(n)$, $i=1, \ldots, N(n)$, then

$$
F_{n}(t, \theta)=\frac{T}{N(n)} F_{d}(t, \theta), \quad 0 \leq t \leq T, \theta \in \mathcal{U}, n=1,2, \ldots,
$$

where $F_{d}$ is given by (42) for the uniform mesh $\left(t_{i}^{(n)}\right)_{i=1, \ldots, N(n)}$. 
For the logistic model (3) we plotted in Figure 72(a) - (c), the generalized sensitivity functions given by (41) with Lebesgue measure $P$ for various values of the final time $T$. For the first value $T=10$ (Figure 72(a)), we note that the GSF curves exhibit "unsettled" shapes, whereas for $T=20$ and $T=50$ (Figure 72(b) and (c)) the shapes stabilize, and common features like minimum, maximum, monotonicity no longer depend on $T$. We attribute the unsettled behavior of the GSF curves for $T=10$ to the fact that the GSF are cumulative functions, and that the information contained in measurements taken in the interval $[0,10]$ on all three parameters can be considerably improved by taking measurements beyond $t=10$. 
On the other hand, starting with approximately $T=20$, the information content relevant to the estimation of our parameters is well contained within $[0, T]$, a fact supported visually by the stabilization of the GSF curves as function of $T$, after we reach $T=20$. As we will see in the next section, the time $T$ where the GSF curves reach their steady shapes, provides a stopping time criterion for the experiments. 
The high correlation between parameters $r$ and $x_{0}$ can be clearly observed in Figure 72(c) and (d), and it is the main cause of the so called "forced-to-one" artifact, thoroughly discussed in [3] and [8]). In order to investigate how the shapes of the GSF curves change in the absence of high correlation, we hold $x_{0}$ constant at its true value 0.1 and consider the new parameter vector $\tilde{\theta}=(K, r)$. The corresponding set of generalized sensitivity functions and their time derivatives for the true values $(17.5,0.7)$ are plotted in Figure 4(a) and (b). We observe immediately that the GSF with respect to $r$ is no longer decreasing; rather, $g s_{r}$ is now increasing even on the time subinterval where it was decreasing when $x_{0}$ was included in the parametrization. The same dramatic change can also be seen when comparing the time derivatives of the GSF curve with respect to $r$ which in the new case no longer has a negative part (see Figures 72(d), and 4(b)). 
On the other hand, we note that the GSF with respect to $K$ (and its corresponding time derivative) remain very similar to the original shape. We explain this based on the fact $K$ had a very low correlation with $x_{0}$ (and also with $r$ ), and therefore is not affected very much by the removal of this parameter.

In conclusion, we note that the decreasing regions of the GSF are directly related to the high correlation between various parameters. These regions are still highly informative when attempting to recover parameters or improve standard errors, and should be included when taking additional data points. 
(a)

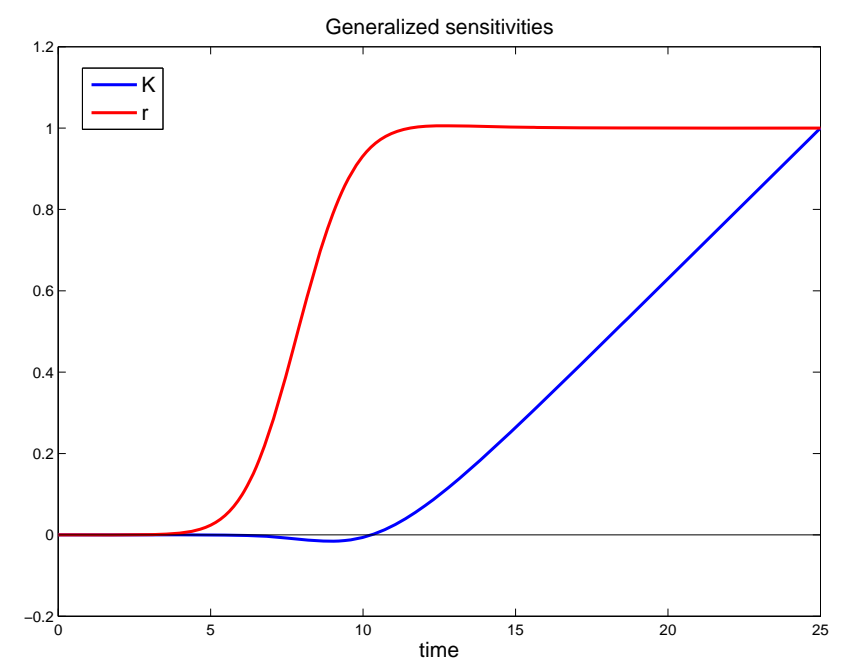

(b)

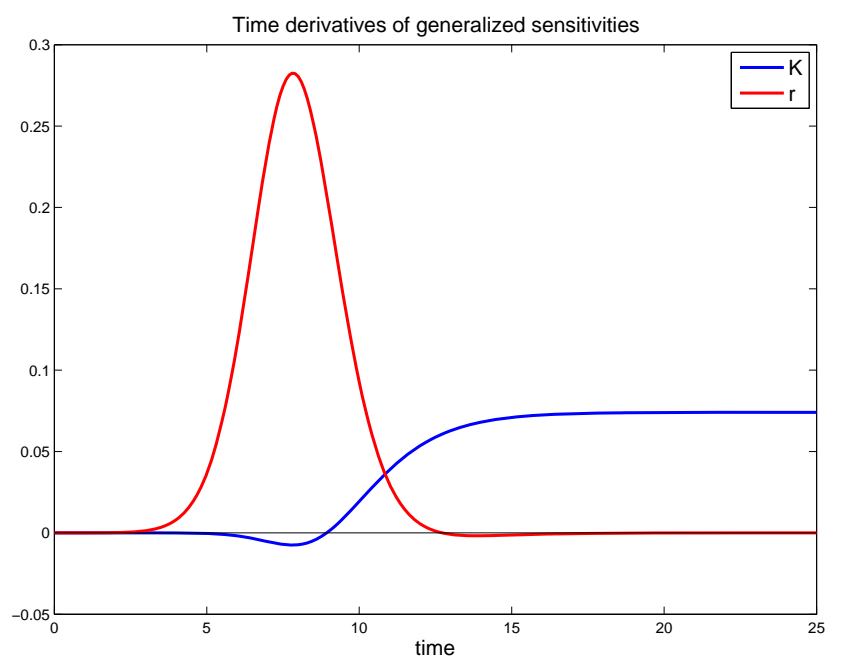

Figure 4: GSFs if only the parameters $\tilde{\theta}=(r, K)$ are considered (panel (a)) and the corresponding time derivatives (panel (b)). The nominal parameters are $\tilde{\theta}_{0}=(17.5,0.7)$ 
As we shall see in the following, the regions of steepest increase and steepest decrease on the graphs of the generalized sensitivity curves correspond to data of high information content with respect to parameter estimation. Intuitively, if one samples additional data points in these regions, one typically obtains more accurate estimates as illustrated by the decrease in magnitude of the corresponding standard errors. 


\section{Theoretical Framework}

- Introduction of the measure $P$ allows for a unified framework optimal design criteria which incorporates many popular design criteria (E-optimal, D-optimal, c-optimal) mentioned .

- Fisher information matrix $F(T, \theta)$ (see $(37)$ ) depends on the measure $P$.

- Here we indicate this dependence by writing $F=F_{P}$-also recall that we can restrict ourselves to probability measures on $[0, T]$.

- Let $\mathcal{P}(0, T)$ denote the set of all probability measures on $[0, T]$ and assume that $\mathcal{J}: \mathbb{R}^{p \times p} \rightarrow \mathbb{R}^{+}$is given. The optimal design problem associated with $\mathcal{J}$ is the problem of finding a probability measure $\hat{P} \in \mathcal{P}(0, T)$ such that 


$$
\mathcal{J}\left(F_{\hat{P}}\left(T, \theta_{0}\right)\right)=\min _{P \in \mathcal{P}(0, T)} \mathcal{J}\left(F_{P}\left(T, \theta_{0}\right)\right) .
$$

- This formulation incorporates all strategies for optimal design which try to optimize a functional depending continuously on the elements of the Fisher information matrix.

- In case of design criteria mentioned earlier, $\mathcal{J}$ is the determinant, the smallest eigenvalue, or a quadratic form, respectively, in terms of the inverse of the Fisher information matrix. 


\section{Existence of optimal sampling strategies}

In order to solve problem (43), one needs a theoretical and computational framework that ensures the existence of a minimizer and provides tractable numerical algorithms to compute it. Unfortunately, the set $\mathcal{P}[0, T]$ does not have a linear space structure (a linear combination of two probability distributions is not a probability distribution) and we cannot take advantage of the plethora of optimization results available in Banach or Hilbert spaces. Hence, we are forced to solve the optimization problem (43) in a metric space setting. Fortunately, probability theory offers a topology which provides the theoretical framework we may utilize. 
With the metric $d(x, y)=|x-y|, x, y \in[0, T]$, the interval $[0, T]$ is a complete, compact and separable metric space. On the set $\mathcal{P}(0, T)$ of Borel probability measures on $[0, T]$ (i.e., probability measures on the Borel subsets of $[0, T]$ ) we introduce the Prohorov metric $\rho$ defined by (see $[14,24,31]$

$$
\rho(P, \tilde{P})=\inf _{A \subset[0, T] \text { closed }}\left\{\epsilon \mid P(A) \leq \tilde{P}\left(A^{\epsilon}\right)+\epsilon\right\}, \quad P, \tilde{P} \in \mathcal{P}(0, T),
$$

where $A^{\epsilon}=\{y \in[0, T] \mid d(y, A) \leq \epsilon\}$. In the following proposition we state properties of the metric space $(\mathcal{P}(0, T), \rho)$ which are inherited from the metric space $([0, T], d)$ and a result on $\rho$-convergence (see $[5,6])$ :

Proposition 0.2. a) The metric space $(\mathcal{P}(0, T), \rho)$ is complete, compact and separable. b) $\rho$-convergence is equivalent to weak*-convergence on $\mathcal{P}(0, T)$ (considering $\mathcal{P}(0, T)$ as a subset of $\left.C_{b}([0, T])^{*}\right)$. 
A consequence of statement b) of this proposition is (see, for instance, $[14,24,30])$

Lemma 0.3. Let $P, P_{n} \in \mathcal{P}(0, T), n=1,2, \ldots$, be given. If $\lim _{n \rightarrow \infty} \rho\left(P_{n}, P\right)=0$ then equivalently

$$
\lim _{n \rightarrow \infty} \int_{0}^{T} g(t) d P_{n}(t)=\int_{0}^{T} g(t) d P(t)
$$

for all continuous functions $g$ on $[0, T]$. 
From the results stated above we obtain immediately the following theorem which is basic to our approach:

Theorem 0.4. Assume that the functional $\mathcal{J}: \mathbb{R}^{p \times p} \rightarrow \mathbb{R}^{+}$is continuous. Then problem (43) has a solution $\hat{P} \in \mathcal{P}(0, T)$.

Proof. The assumption on $\mathcal{J}$ and the lemma imply that the mapping $P \rightarrow \mathcal{J}\left(F_{P}\left(T, \theta_{0}\right)\right)$ is continuous on $\mathcal{P}(0, T)$. Then the result follows from compactness of $\mathcal{P}(0, T)$. 


\section{Approximation Issues}

- Having argued the existence of a solution problem (43), we next turn our attention to the computational aspects related to finding $\hat{P}$.

- Note that $(\mathcal{P}(0, T), \rho)$ is an infinite dimensional metric space, so we need to construct finite dimensional approximations $\hat{P}_{n}$ for $\hat{P}$ which to converge to $\hat{P}$ in the Prohorov metric when $n \rightarrow \infty$.

- The following density result (formulated and proven for a general set $Q$ in [4]) provides us the needed structure to build such finite dimensional approximations. We state here the result for $Q=[0, T]$. 
Theorem 0.5. Let $Q_{0}=\left\{t_{j}\right\}_{j=1}^{\infty}$ be a countable, dense subset of $[0, T]$ and $\delta_{t_{j}}$ be the Dirac measure with atom at $t_{j}$. Then the set

$$
\begin{aligned}
\mathcal{P}_{0}(0, T):=\{P \in \mathcal{P}(0, T) \mid P & =\sum_{j=1}^{k} p_{j} \delta_{t_{j}}, k \in \mathbb{N}^{+}, \\
t_{j} & \left.\in Q_{0}, p_{j} \geq 0, p_{j} \text { rational, } \sum_{j=1}^{k} p_{j}=1\right\}
\end{aligned}
$$

(i.e., the set of $P \in \mathcal{P}(0, T)$ with finite support in $Q_{0}$ and rational masses) is dense in $(\mathcal{P}(0, T), \rho)$ in the Prohorov metric $\rho$. 
Let $Q_{d}$ be a dense subset of $[0, T]$-theorem above simply says that any probability distribution in $\mathcal{P}(0, T)$ can be approximated arbitrarily closely in the Prohorov metric by finite convex combinations of Dirac measures supported in $Q_{d}$. Given $Q_{d}=\bigcup_{M=1}^{\infty} Q_{M}$ with $Q_{M}=\left\{t_{j}^{M}\right\}_{j=1, \ldots, M}$ chosen such that $Q_{d}$ is dense in $[0, T]$, we define, for $M=1,2, \ldots$,

$$
\begin{aligned}
\mathcal{P}^{M}(0, T):=\left\{P \in \mathcal{P}(0, T) \mid P=\sum_{j=1}^{M} p_{j} \delta_{t_{j}^{M}}, t_{j}^{M} \in Q_{M}, p_{j} \geq 0,\right. \\
\left.p_{j} \text { rational, } \sum_{j=1}^{M} p_{j}=1\right\},
\end{aligned}
$$

which is a compact subset of $(\mathcal{P}(0, T), \rho)$. 
Then we have that " $\mathcal{P}^{M}(0, T) \rightarrow \mathcal{P}(0, T)$ " in the $\rho$-topology; that is, elements in the infinite dimensional space $\mathcal{P}(0, T)$ can be approximated arbitrarily closely by elements from the finite dimensional space $\mathcal{P}^{M}(0, T)$ for $M$ sufficiently large. In particular the minimizer $\hat{P}$ of $\mathcal{J}\left(F_{P}\left(T, \theta_{0}\right)\right)$ over $\mathcal{P}(0, T)$ can be approximated by a sequence of minimizers of $\mathcal{J}\left(F_{P}\left(T, \theta_{0}\right)\right)$ over the finite dimensional spaces $\mathcal{P}^{M}(0, T)$ as summarized by the following theorem:

Theorem 0.6. Let $\mathcal{P}^{M}(0, T), M=1,2, \ldots$, be defined as above. Suppose $\hat{P}_{M}$ is minimizer for $\mathcal{J}\left(F_{P}\left(T, \theta_{0}\right)\right)$ over $\mathcal{P}^{M}(0, T)$, i.e.,

$$
\hat{P}_{M}=\underset{P \in \mathcal{P}^{M}(0, T)}{\operatorname{argmin}} \mathcal{J}\left(F_{P}\left(T, \theta_{0}\right)\right), \quad M=1,2, \ldots .
$$

Then we have $\rho\left(\hat{P}_{M}, \hat{P}\right) \rightarrow 0$ as $M \rightarrow \infty$. 
The following corollary is an immediate consequence of this theorem: Corollary 0.7. Assume that $\mathcal{J}: \mathbb{R}^{p \times p} \rightarrow \mathbb{R}^{+}$is continuous and let

$$
\hat{P}=\underset{P \in \mathcal{P}(0, T)}{\operatorname{argmin}} \mathcal{J}\left(F_{P}\left(T, \theta_{0}\right)\right) .
$$

For $n=1,2, \ldots$ we set $\Delta t=T / n, t_{i}^{(n)}=i \Delta t, i=0, \ldots, n$, and define $\mathcal{P}_{n}(0, T) \subset \mathcal{P}(0, T)$ by

$$
\begin{array}{r}
\mathcal{P}_{n}(0, T)=\left\{P_{n}=\sum_{j=0}^{n} \mu_{j}^{(n)} \delta_{t_{j}^{(n)}}, \mu_{j}^{(n)} \text { rational, } \mu_{j}^{(n)} \geq 0\right. \\
\text { and } \left.\sum_{j=0}^{n} \mu_{j}^{(n)}=1\right\}, n=1,2, \ldots,
\end{array}
$$

where $\delta_{t_{j}^{(n)}}$ denotes the Dirac measure supported at $t_{j}^{(n)}$. 
Furthermore, let

$$
\hat{P}_{n}=\underset{P_{n} \in \mathcal{P}_{n}(0, T)}{\operatorname{argmin}} \mathcal{J}\left(F_{P_{n}}\left(T ; \theta_{0}\right)\right) .
$$

Then we have

$$
\begin{aligned}
\lim _{n \rightarrow \infty} \rho\left(\hat{P}_{n}, \hat{P}\right) & =0, \\
\lim _{n \rightarrow \infty} \mathcal{J}\left(F_{\hat{P}_{n}}\left(T, \theta_{0}\right)\right) & =\mathcal{J}\left(F_{\hat{P}}\left(T, \theta_{0}\right)\right) .
\end{aligned}
$$


Proof. Existence of $\hat{P}$ and $\hat{P}_{n}, n=1,2, \ldots$, follows from Theorem 0.4. It is easy to see that $Q=\bigcup_{n=1}^{\infty} Q_{n}$ with $Q_{n}=\left\{t_{j}^{(n)} \mid j=0, \ldots, n\right\}$ is dense in $[0, T]$. Then (46) follows immediately from Theorem 0.6. Continuity of $P \rightarrow \mathcal{J}\left(F_{P}\left(T, \theta_{0}\right)\right)$ (compare Lemma 0.3) and (46) imply (47). 


\section{Choosing a Final Time $T$}

\section{Eigenvalues of the Fisher Information Matrix}

As we have seen before, the Fisher information matrix relates the variation in the parameter estimates to variations in the measurements. Also, its inverse $F^{-1}$ is used to define the generalized sensitivity functions (41). Due to these facts, the condition number of FIM is expected to play an important role in the statistical properties of $\hat{\theta}$, and numerical stability issues suggest that it is important to choose $T$ such that the Fisher information matrix is well conditioned. In this subsection we present a theoretical result which shows that the minimum and maximum eigenvalues of the Fisher information matrix, as well as its spectral norm are monotonically increasing functions of the final time $T$. As we shall 
see in the next subsection, in the particular case where the condition number of the FIM is decreasing, which is true for the logistic model, we can use the condition number of the FIM to indicate an appropriate stopping time $T$. In order to simplify notation we do not indicate the dependence of the FIM and of quantities associated with the FIM on $\theta$ in this subsection. 
Theorem 0.8. Assume that $\operatorname{det} F(T) \neq 0$. Then $\operatorname{det} F(T+\Delta) \neq 0$ for all $\Delta \geq 0$ and

i) the minimum and the maximum eigenvalues of the Fisher information matrix are increasing functions of the final time $T$, i.e.,

$\lambda_{\min }(T) \leq \lambda_{\min }(T+\Delta) \quad$ and $\quad \lambda_{\max }(T) \leq \lambda_{\max }(T+\Delta), \quad \Delta \geq 0$,

ii) the spectral norm of the covariance matrix $F(T)^{-1}$ is a non-increasing function of the final time $T$, i.e.,

$$
\left\|F(T+\Delta)^{-1}\right\|_{2} \leq\left\|F(T)^{-1}\right\|_{2}, \quad \Delta \geq 0
$$

(here $\|\cdot\|_{2}$ denotes the spectral norm of a matrix). 
Proof. i) The matrix $F(T)$ is positive definite. If $\lambda_{\min }(T)$ and $\lambda_{\max }(T)$ denote the smallest and the largest eigenvalue of $F(T)$, then we have

$$
\lambda_{\min }(T)\|a\|_{2}^{2} \leq a^{\top} F(T) a \leq \lambda_{\max }(T)\|a\|_{2}^{2}, \quad a \in \mathbb{R}^{p} .
$$

For $a \in \mathbb{R}^{p}$ and $\Delta>0$ we have

$$
\begin{aligned}
\lambda_{\min }(T)\|a\|^{2}+ & \int_{T}^{T+\Delta} \frac{1}{\sigma^{2}(t)}\left\|\nabla_{\theta} f\left(t, \theta_{0}\right) a\right\|^{2} d P(t) \leq a^{\top} F(T+\Delta) a \\
& \leq \lambda_{\max }(T)\|a\|^{2}+\int_{T}^{T+\Delta} \frac{1}{\sigma^{2}(t)}\left\|\nabla_{\theta} f\left(t, \theta_{0}\right) a\right\|^{2} d P(t) .
\end{aligned}
$$

The left side of this inequality implies

$$
\lambda_{\min }(T) \leq \lambda_{\min }(T+\Delta) .
$$

If we choose $a$ as an eigenvector of $F(T)$ corresponding to $\lambda_{\max }(T)$ 
then we obtain

$$
\begin{aligned}
& a^{\top} F(T+\Delta) a= \\
& \qquad \begin{aligned}
\lambda_{\max }(T)\|a\|^{2}+\int_{T}^{T+\Delta} \frac{1}{\sigma^{2}(t)} & \left\|\nabla_{\theta} f\left(t, \theta_{0}\right) a\right\|^{2} d P(t) \\
& \leq \lambda_{\max }(T+\Delta)\|a\|^{2},
\end{aligned}
\end{aligned}
$$

and from the right side of this inequality we obtain

$$
\lambda_{\max }(T) \leq \lambda_{\max }(T+\Delta) .
$$

ii) The largest and smallest eigenvalues of $F^{-1}(T+\Delta)$ are respectively $1 / \lambda_{\min }(T+\Delta)$ and $1 / \lambda_{\max }(T+\Delta)$. We note that $\left\|F(T+\Delta)^{-1}\right\|_{2}=1 / \lambda_{\min }(T+\Delta)$, since $F(T+\Delta)^{-1}$ is symmetric and the spectral radius of $\left(F^{-1}(T+\Delta)\right)^{\top} F^{-1}(T+\Delta)$ is $\lambda_{\min }^{-2}(T+\Delta)$. Then $(48)$ implies that $\operatorname{det} F(T+\Delta) \neq 0$, and ii) follows immediately from (48). 
Remark 0.9. The statements at the beginning of this subsection indicate that it would be desirable to show that the condition number $\rho_{F}(T):=\lambda_{\max }(T) / \lambda_{\min }(T)$ of the Fisher information matrix is a non increasing function of the final time $T$, i.e.,

$$
\rho_{F}(T) \geq \rho_{F}(T+\Delta) .
$$

Fortunately, this is true for the logistic model, where it is the consequence of the fact that the minimum eigenvalue increases at a significantly faster rate than the maximum eigenvalue (see Table 1 and Figure 5(a)). We also note that although both the minimum and the maximum eigenvalues increase, the rates at which they increase are directly related to the first order traditional sensitivity functions. This can be readily seen for example from (49) which provides some measure of the gap between $\lambda_{\max }(T)$ and $\lambda_{\max }(T+\Delta)$. 


\begin{tabular}{|c|r|c|c|}
\hline$T$ & $\lambda_{\min }(T)$ & $\lambda_{\max }(T)$ & \multicolumn{1}{c|}{$\rho_{F}(T)$} \\
\hline \hline 15 & 2.6112 & 11403.3913 & 4367.0612 \\
20 & 7.3132 & 11404.9422 & 1559.5081 \\
25 & 12.2328 & 11404.9461 & 932.3246 \\
30 & 17.1568 & 11404.9467 & 664.7493 \\
\hline
\end{tabular}

Table 1: Values for $\lambda_{\min }(T), \lambda_{\max }(T)$ and the condition number of the Fisher information matrix corresponding to the logistic model, as the final time $T$ increases. The nominal parameter vector is $\theta_{0}=$ $(17.5,0.7,0.1)$.

However, although desirable, the relation (50) is NOT true in 
general. To see this we consider the initial value problem

$$
\dot{x}(t)=-\mu_{1} e^{-t}+\frac{\mu_{2}}{1+t}, \quad x(0)=\mu_{1},
$$

for positive $t$, whose solution is

$$
x\left(t, \mu_{1}, \mu_{2}\right)=\mu_{1} e^{-t}+\mu_{2} \ln (1+t), \quad t \geq 0 .
$$

We take $f\left(t, \mu_{1}, \mu_{2}\right)=x\left(t, \mu_{1}, \mu_{2}\right)$ and observe that the Fisher information matrix does not depend on the parameters in this case, because $f$ is a linear function of the parameters. Of course, the eigenvalues of the Fisher information matrix are increasing as required by Theorem 0.8 (see also Table 2), however the largest eigenvalue increases much faster than the smallest one, so that the condition number is also increasing. The measure $P$ was taken to be the Lebesgue measure. This example shows, that in general the condition number of the Fisher information matrix is NOT a decreasing function of the final time $T$. 


\begin{tabular}{|c|r|r|r|}
\hline$T$ & $\lambda_{\min }(T)$ & $\lambda_{\max }(T)$ & \multicolumn{1}{c|}{$\rho_{F}(T)$} \\
\hline \hline 10 & 0.4882 & 30.5071 & 62.4949 \\
15 & 0.4944 & 64.2787 & 130.0073 \\
20 & 0.4967 & 106.2849 & 215.0086 \\
25 & 0.4977 & 156.5763 & 314.5862 \\
30 & 0.4983 & 212.6548 & 426.7402 \\
\hline
\end{tabular}

Table 2: Values for $\lambda_{\min }(T), \lambda_{\max }(T)$ and the condition number of the Fisher information matrix corresponding to the model (51) for increasing values of the final time $T$. 
(a) $\log _{10}$ condition number of FIM

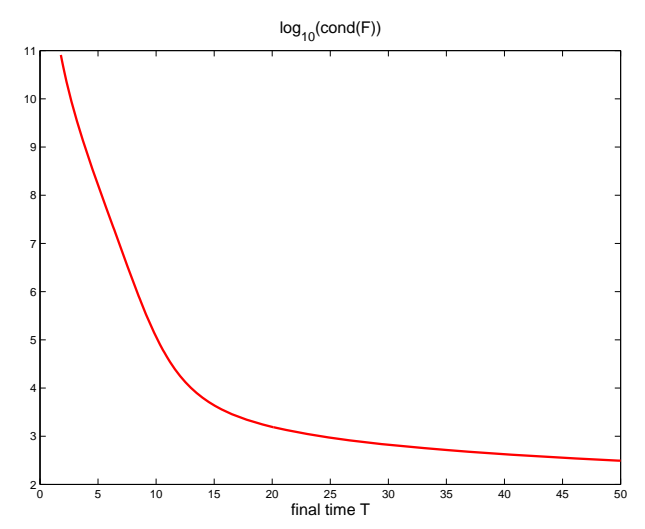

(c) $2^{\text {nd }}$ derivative: $\log _{10}$ cond(FIM)

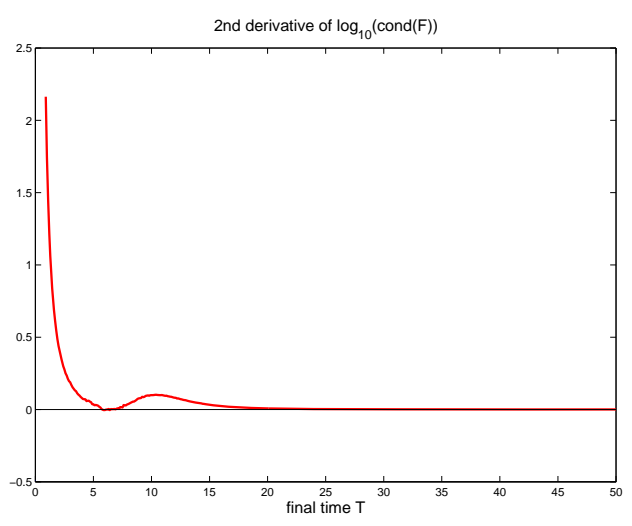

(b) $1^{\text {st }}$ derivative: $\log _{10}$ cond(FIM)

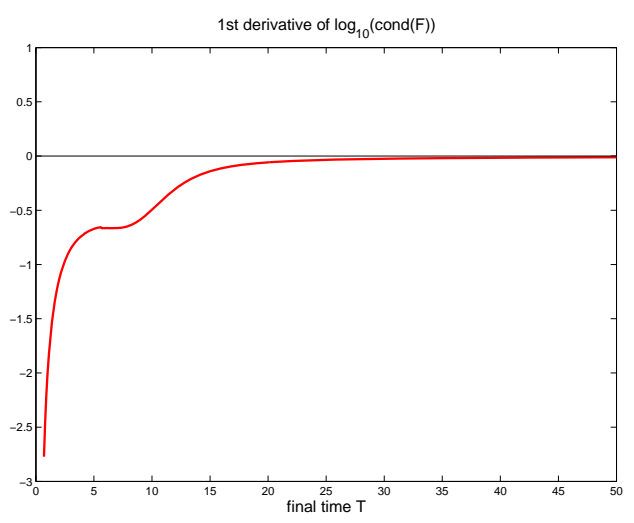

(d) correlation coefficients

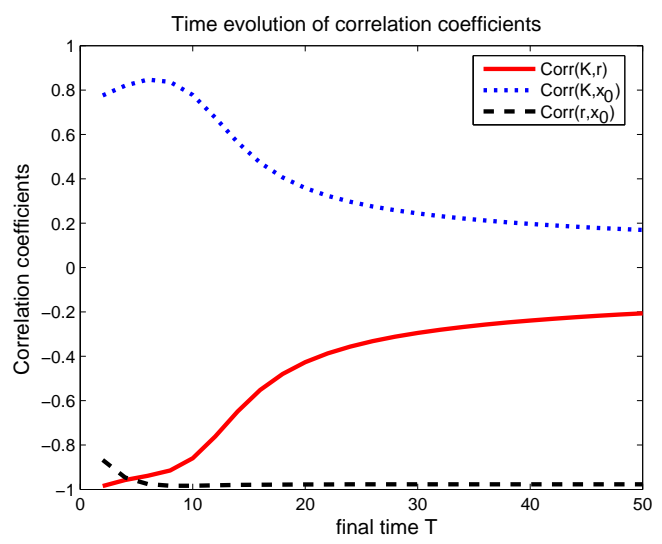

Figure 5: 
Topics covered in [9], Generalized sensitivities and optimal

experimental design, H. T. Banks, Sava Dediu, Stacey L. Ernstberger and Franz Kappel, November, 2009-see also CRSC-TR08-12, September, 2008-include computational illustrations on

- Tools for Choosing a Final Time $\boldsymbol{T}$

- Sensitivity Functions

- Condition Number of the Fisher Information Matrix

- Choosing a Sampling Distribution in $[\mathbf{0}, \boldsymbol{T}]$

- Choosing the Size of a Sampling Distribution

- Sensitivity Functions as Design Tools - Numerical Experiments 


\section{Using Optimal Design Criteria}

In this subsection we advocate a new optimal design criterion which is based on the idea of finding a measure $P$ of the form (8), i.e.,

$$
P=\sum_{i=1}^{n} \delta_{t_{i}},
$$

which provides the best discrete approximation for the FIM $F_{C}$ obtained with $P=\mu$, where $\mu$ is the Lebesgue measure on $[0, T]$. The FIM corresponding to $P$ is denoted by $F_{D}$. In other words, we want to find the optimal time distribution $t_{1}<t_{2}<\cdots<t_{n}$ in $[0, T]$ such that

$$
F_{D}^{n}=\sum_{j=1}^{n} \frac{1}{\sigma^{2}\left(t_{j}\right)} \nabla_{\theta} f\left(t_{j}, \theta_{0}\right)^{\top} \nabla_{\theta} f\left(t_{j}, \theta_{0}\right)
$$


best approximates

$$
F_{C}=\int_{0}^{T} \frac{1}{\sigma^{2}(s)} \nabla_{\theta} f\left(s, \theta_{0}\right)^{\top} \nabla_{\theta} f\left(s, \theta_{0}\right) d s .
$$

This design criterion is motivated by the intuitive notion that the best case scenario we can possible have when estimating the parameter $\theta_{0}$ is the one when we have available all the measurements at all times in the interval $[0, T]$. The natural approach when we have a limited number of observations is therefore to find the sampling distribution which gives the best discrete approximation for $F_{C}$. By approximation we means approximation with respect to the Frobenius norm of matrices, i.e., we use the design functional

$$
\mathcal{J}_{1}\left(F_{D}^{n}\right)=\sum_{i=1}^{p} \sum_{j=1}^{p}\left|\left(F_{C}\right)_{i j}-\left(F_{D}^{n}\right)_{i j}\right|^{2},
$$


For comparison we also use the design functional

$$
\mathcal{J}_{2}(F)=\sum_{i=1}^{p} \frac{1}{\theta_{0, i}^{2}}\left(F^{-1}\right)_{i i} .
$$

This functional is the sum, over all components of the parameter vector, of the squared normalized standard errors for the components of the parameter vector and can be defined for general probability measures $P$. 
(a) $S E(K)$

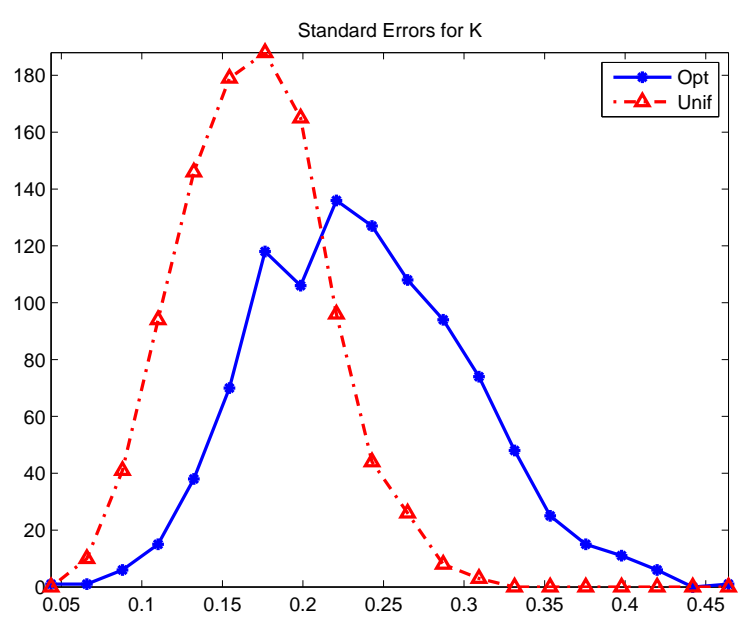

(c) $S E\left(x_{0}\right)$

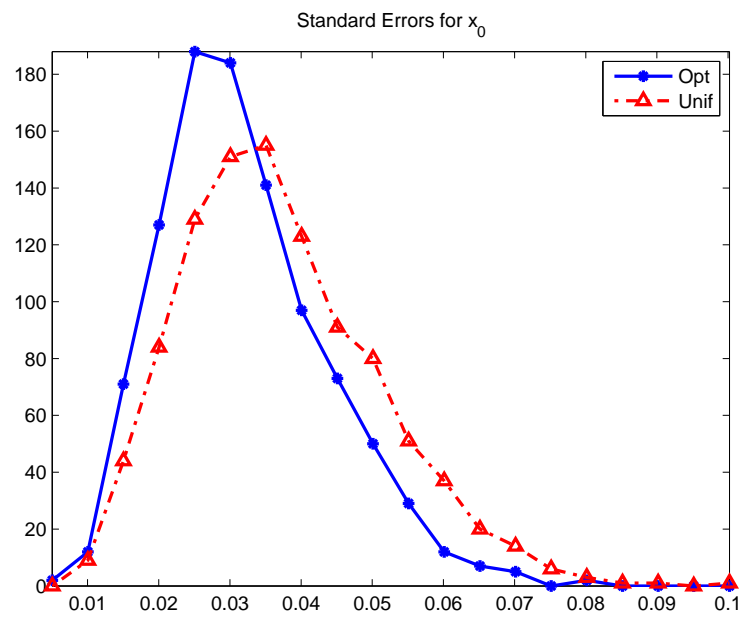

(b) $S E(r)$

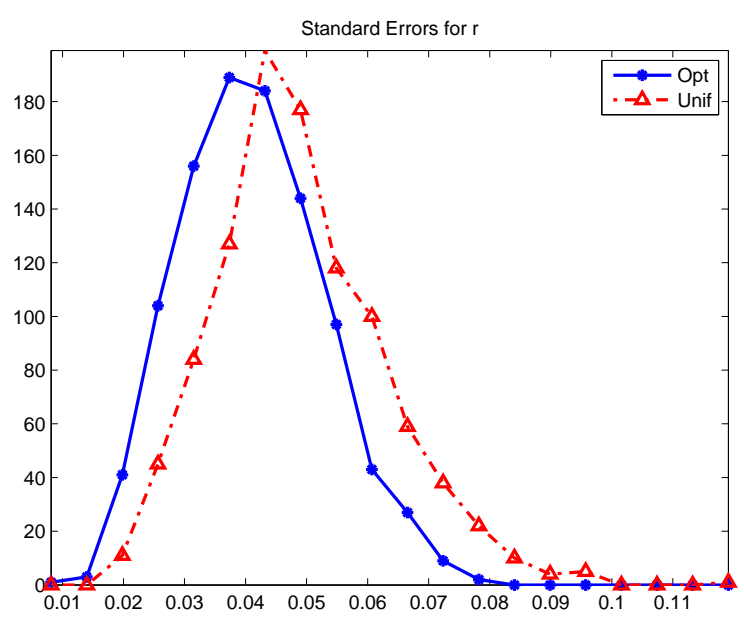

(d) distribution of time points

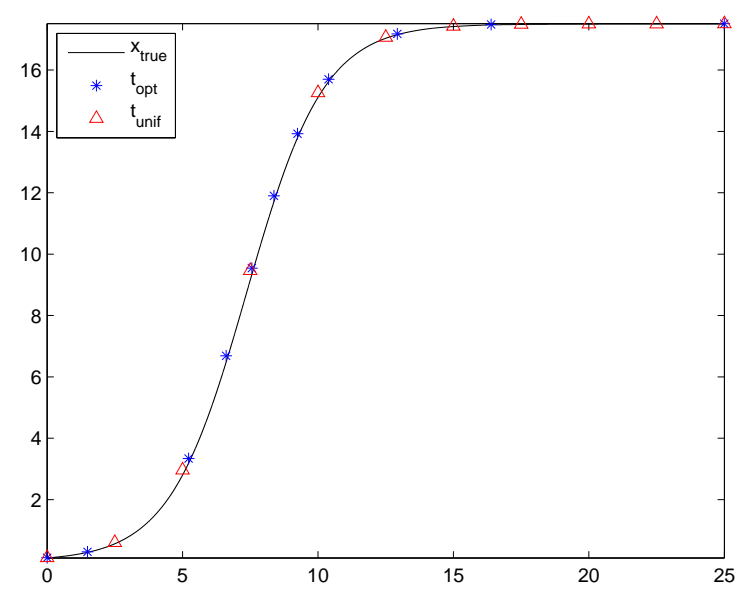

Figure 6: Comparison of optimal ${ }_{1}\left(\right.$ ddsing $\left._{8} \mathcal{J}_{1}\right)$ versus uniform sampling 


\section{Numerical Simulations}

We consider two inverse problems:

1. Assume that we know true parameter value $\theta_{0}$-seek to optimize placement of time points for data collection - optimization criterion: first minimize $J_{1}$-to optimize placement of the time points $t_{i}$, choose $n$ positive numbers $\mu_{i}, i=1, \ldots, n$, such that $\sum_{i=1}^{n} \mu_{i}=T$, where $T$ is the final time-time points are established as $t_{1}=0$ and $t_{i}=t_{i-1}+\mu_{i-1}, i=2, \ldots, n+1$-initially chose $\mu_{i}=T / n$, creating a uniformly spaced time distribution, $t_{\text {unif }}$. Then we use fmincon, a constrained gradient-based optimization algorithm in MATLAB, to return an optimal $\mu$-vector, which in turn produces an optimal $t$-vector, denoted by $t_{\mathrm{opt}}$-note that this will produce an optimal mesh among those meshes containing both 0 and $T$; 
however, may not be optimal among all possible sampling distributions including those in which 0 or $T$ or both may be missing.

2. Then considered a parameter estimation problem which compares the effectiveness of $t_{\text {unif }}$ versus $t_{\text {opt }}$. We created noisy data $y(t)$ by generating the logistic curve using the true parameter vector $\theta_{0}$ and then adding Gaussian noise with $\sigma_{0}^{2}=0.16$. Then we solve two parameter estimation problems (one using only the points in $t_{\text {unif }}$, and the other using only the points in $t_{\text {opt }}$ ) to determine which grid best recovers $\theta$. We use the least squares functional

$$
J(\theta)=\sum_{i=1}^{n+1}\left|y\left(t_{i}\right)-f\left(t_{i}, \theta\right)\right|^{2}
$$

to determine the optimal $\theta$ in each case-also considered the corresponding standard errors. 
For these simulations we use $n=10, \theta_{0}=(17.5,0.7,0.1)$ and $T=25$. We run the first inverse problem one time for the cost functional (54) to obtain an optimal sampling distribution. We then run the second inverse problem (for (56)) 1000 times (using an initial estimate $\left.\theta^{0}=1.4 \cdot \theta_{0}\right)$, and compute the average $\hat{\theta}^{n}$ and SE-values over the total number of simulations. The resulting standard errors for each parameter can be seen in Figure 6(a) - (c). Then in Figure 6(d), we depict the logistic curve plotted using the true $\theta_{0}$, along with the time point location for both $t_{\text {unif }}$ and $t_{\mathrm{opt}}$. The average $\theta$ value over these 1000 runs is $(17.523,0.69728,0.10743)$, and the average standard errors are approximately $(0.24,0.042,0.033)$. 
(a) $S E(K)$

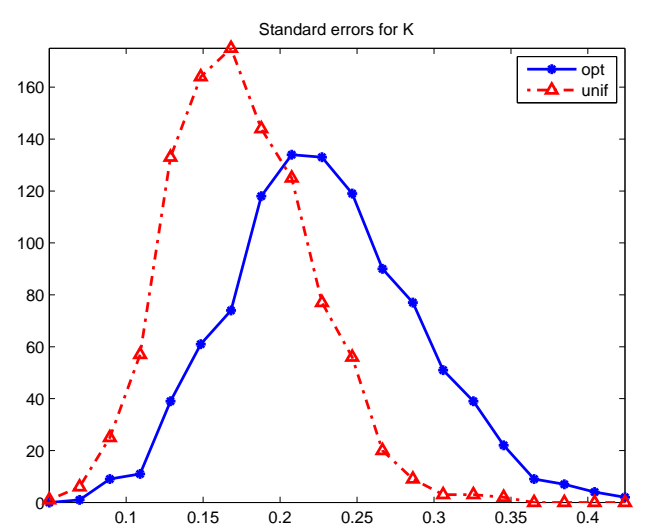

(c) $S E\left(x_{0}\right)$

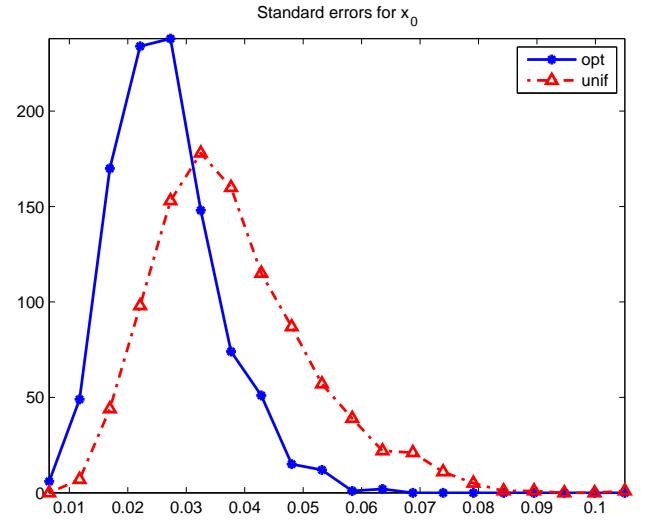

(b) $S E(r)$

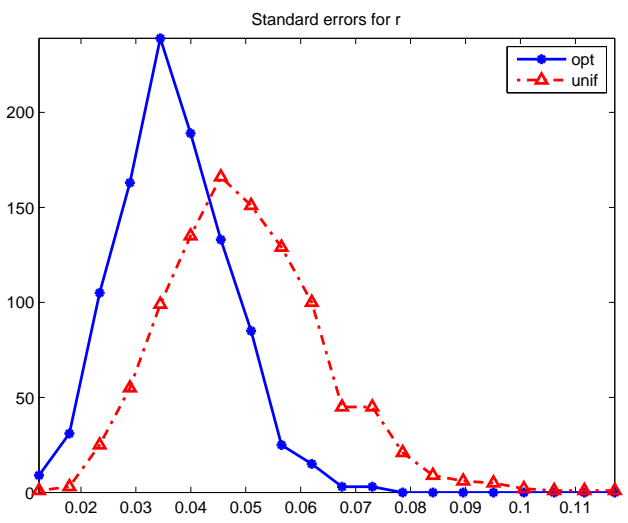

(d) distribution of time points

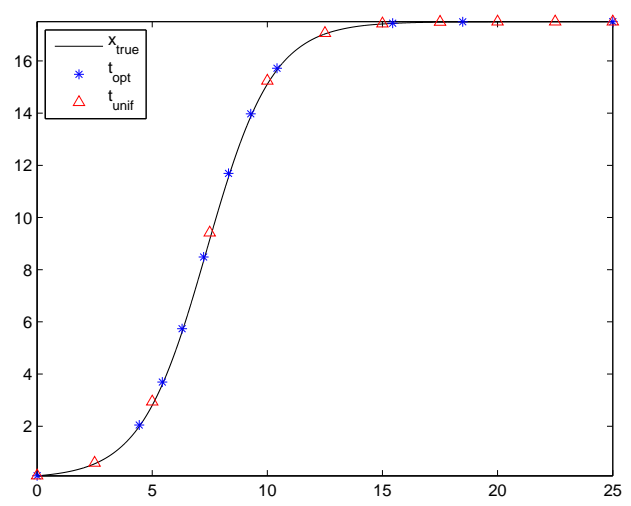

Figure 7: Comparison of optimal (using $J_{2}$ ) versus uniform 
Next, we run the first inverse problem one time for the cost functional (55). We then run the second inverse problem 1000 times (using an initial estimate $\theta^{0}=1.4 \cdot \theta_{0}$ ), and compute the average $\hat{\theta}^{n}$ and $\mathrm{SE}$ values over the total number of simulations. The resulting standard errors for each parameter can be seen in Figure 7(a) - (c). Then in Figure 7(d), we plot the logistic curve using the true $\theta_{0}$, along with the time point locations for each of $t_{\text {unif }}$ and $t_{\mathrm{opt}}$. The average $\theta$ value over these 1000 runs is $(17.5062,0.6992,0.1045)$, and the average standard errors are approximately $(0.23,0.037,0.027)$.

Overall, it appear that for this example the second cost functional (55), which considers the standard errors as opposed to the continuous Fisher information matrix, performs slightly better (although both functionals perform adequately). 


\section{References}

[1] R. C. Aster, B. Borchers and C. H. Thurber, Parameter Estimation and Inverse Problems, Elsevier Academic Press, New York, NY, 2005.

[2] H. M. Adelman and R. T. Haftka, Sensitivity analysis of discrete structural systems, A.I.A.A. Journal, 24 (1986), 823 - 832.

[3] P. Bai, H. T. Banks, S. Dediu, A. Y. Govan, M. Last, A. Loyd, H. K. Nguyen, M. S. Olufsen, G. Rempala and B. D. Slenning, Stochastic and deterministic models for agricultural production networks, Math. Biosci. and Engineering, 4 (2007), 373 - 402.

[4] H. T. Banks and K. L. Bihari, Modeling and estimating uncertainty in parameter estimation, Inverse Problems, 17 (2001), $95-111$. 
[5] H. T. Banks and D. M. Bortz, Inverse problems for a class of measure dependent dynamical systems, CRSC-TR04-33, NCSU, September, 2004; J. Inverse and Ill-posed Problems, 13 (2005), $103-121$.

[6] H. T. Banks, D. M. Bortz, G. A. Pinter and L. K. Potter, Modeling and imaging techniques with potential for application in bioterrorism, Chapter 6 in Bioterrorism: Mathematical Modeling Applications in Homeland Security, (H.T. Banks and C. Castillo-Chavez, eds.), Frontiers in Applied Mathematics FR28, SIAM, Philadelphia, 2003, pp. 129-154.

[7] H. T. Banks, M. Davidian, J. R. Samuels Jr., and K. L. Sutton, An inverse problem statistical methodology summary, CRSC-TR08-01, NCSU, January, 2008; Chapter 11 in Statistical Estimation Approaches in Epidemiology, (edited by Gerardo Chowell, Mac Hyman, Nick Hengartner, Luis M.A Bettencourt 
and Carlos Castillo-Chavez), Springer, Berlin Heidelberg New York, 2009, pp. 249-302.

[8] H. T. Banks, S. Dediu and S.L. Ernstberger, Sensitivity functions and their uses in inverse problems, J. Inv. Ill-posed Problems, 15 (2007), $683-708$.

[9] H.T. Banks, Sava Dediu, Stacey L. Ernstberger and Franz Kappel, A new approach to optimal design problems, CRSC-TR08-12, September, 2008; Revised, November, 2009

[10] H. T. Banks, S. Dediu and H. K. Nguyen, Sensitivity of dynamical systems to parameters in a convex subset of a topological vector space, Math. Biosci. and Engineering, 4 (2007), $403-430$.

[11] H. T. Banks, S. L. Ernstberger and S. L. Grove, Standard errors and confidence intervals in inverse problems: sensitivity and 
associated pitfalls, J. Inv. Ill-posed Problems, 15, (2006), 1 - 18.

[12] J. J. Batzel, F. Kappel, D. Schneditz and H. T. Tran, Cardiovascular and Respiratory Systems: Modeling, Analysis and Control, Frontiers in Applied Mathematics FR34, SIAM, Philadelphia, 2006.

[13] M. P. F. Berger and W. K. Wong (Editors), Applied Optimal Designs, John Wiley \& Sons, Chichester, UK, 2005.

[14] P. Billingsley, Convergence of Probability Measures, John Wiley \& Sons, New York, NY, 1968.

[15] G. Casella and R. L. Berger, Statistical Inference, Duxbury, California, 2002.

[16] J. B. Cruz, (Editor), System Sensitivity Analysis, Dowden, Hutchinson \& Ross, Inc., Stroudsberg, PA, 1973. 
[17] M. Davidian and D. Giltinan, Nonlinear Models for Repeated Measurement Data, Chapman \& Hall, London, 1998.

[18] J. Dieudonné, Foundations of Modern Analysis, Academic Press, New York, 1960.

[19] M. Eslami, Theory of Sensitivity in Dynamic Systems: An Introduction, Springer-Verlag, Berlin, 1994.

[20] V. V. Fedorov, Theory of Optimal Experiments, Academic Press, New York and London, 1972.

[21] V. V. Fedorov and P. Hackel, Model-Oriented Design of Experiments, Springer-Verlag, New York, NY, 1997.

[22] P. M. Frank, Introduction to System Sensitivity Theory, Academic Press, New York, NY, 1978.

[23] A. R. Gallant, Nonlinear Statistical Models, John Wiley \& Sons, Inc., New York, NY, 1987. 
[24] P. J. Huber, Robust Statistics, John Wiley \& Sons, Inc., New York, NY, 1981.

[25] R. I. Jennrich, Asymptotic properties of non-linear least squares estimators, Ann. Math. Statist., 40 (1969), 633 - 643.

[26] F. Kappel and M. Munir, A New Approach to Optimal Design Problems, Proc. Internatl. Conf. Nonlinear Analysis and Optimization, October 6 - 10, 2008, Budva (Montenegro), to appear.

[27] M. Kleiber, H. Antunez, T. D. Hien and P. Kowalczyk, Parameter Sensitivity in Nonlinear Mechanics: Theory and Finite Element Computations, John Wiley \& Sons, New York, NY, 1997.

[28] M. Kot, Elements of Mathematical Ecology, Cambridge University Press, Cambridge, 2001, p. 7 - 9. 
[29] D. G. Luenberger, Optimization by Vector Space Methods, John Wiley \& Sons, New York, NY, 1969.

[30] V. Paulauskas and A. Račkauskas, Approximation Theory in the Central Limit Theorem, Kluwer Academic Publ., Dordrecht, 1989 .

[31] Yu. V. Prohorov, Convergence of random processes and limit theorems in probability theory, Theor. Prob. Appl., 1 (1956), $157-214$.

[32] F. Pukelsheim, Optimal Design of Experiments, John Wiley \& Sons, New York, NY, 1993.

[33] A. Saltelli, K. Chan and E. M. Scott, eds., Sensitivity Analysis, Wiley Series in Probability and Statistics, John Wiley \& Sons, New York, NY, 2000.

[34] G. A. F. Seber and C. J. Wild, Nonlinear Regression, John 
Wiley \& Sons, New York, NY, 1989.

[35] K. Thomaseth and C. Cobelli, Generalized sensitivity functions in physiological system identification, Ann. Biomed. Eng., 27(5) (1999), $607-616$. 